\title{
REVIEW ARTICLE Aryl hydrocarbon receptor and intestinal immunity
}

\author{
Bruno Lamas ${ }^{1,2}$, Jane M. Natividad ${ }^{2}$ and Harry Sokol ${ }^{1,2}$
}

\begin{abstract}
Aryl hydrocarbon receptor (AhR) is a member of the basic helix-loop-helix-(bHLH) superfamily of transcription factors, which are associated with cellular responses to environmental stimuli, such as xenobiotics and oxygen levels. Unlike other members of bHLH, AhR is the only bHLH transcription factor that is known to be ligand activated. Early AhR studies focused on understanding the role of AhR in mediating the toxicity and carcinogenesis properties of the prototypic ligand 2,3,7,8-tetrachlorodibenzo- $p$-dioxin (TCDD). In recent years, however, it has become apparent that, in addition to its toxicological involvement, AhR is highly receptive to a wide array of endogenous and exogenous ligands, and that its activation leads to a myriad of key host physiological functions. In this study, we review the current understanding of the functions of AhR in the mucosal immune system with a focus on its role in intestinal barrier function and intestinal immune cells, as well as in intestinal homeostasis.
\end{abstract}

Mucosal Immunology (2018) 11:1024-1038; https://doi.org/10.1038/s41385-018-0019-2

\begin{abstract}
AHR SIGNALING PATHWAY
Aryl hydrocarbon receptor (AhR) is widely expressed by different cell types throughout the body. ${ }^{1-4}$ Numerous genes are regulated by $A h R$, including those that encode xenobiotic metabolizing enzymes, such as Cytochrome P450 1A1 (Cyp1a1). ${ }^{5,6}$ The current model of canonical AhR cellular signaling is based on induction of the Cypla1 gene by AhR (Fig. 1). ${ }^{7,8}$ In its inactive state, AhR is present in the cytoplasm and exists as a multi-protein complex with two chaperone proteins, heat shock protein (Hsp) 90 and the co-chaperone p23. ${ }^{9}$ Upon binding to a ligand, the AhR complex undergoes a conformational change and subsequently translocates into the nucleus, where $A h R$, along with its ligand, is released from the rest of the chaperone proteins. ${ }^{10}$ The remaining AhR:ligand complex heterodimerizes with its nuclear partner, Arnt. The AhR:ligand:Arnt complex eventually binds to the genomic region containing its specific DNA recognition site, called the dioxin response element (DRE), which is situated upstream of the Cyp1a1 and AhR repressor (AhRR) genes ${ }^{8,11,12}$ (Fig. 1a). The canonical AhR pathway also leads to the transcription of additional phase I xenobiotic metabolizing enzymes, such as Cyp1a2 and Cyp1b1, 8,11,12 as well as phase II enzymes, such as glutathione-S-transferase $A_{1}{ }^{13,14} \mathrm{NAD}(\mathrm{P}) \mathrm{H}$ : quinone oxidoreductase $1,{ }^{15}$ uridine $5^{\prime}$-diphosphate-glucuronosyltransferase $1 A^{16}$ and aldehyde dehydrogenase $3^{17}$ (Fig. 1a). These phase I and II enzymes have a role in the negative feedback loop of AhR signaling through metabolic depletion of the AhR ligand pool.

AhR has been characterized as a promiscuous receptor because of its propensity to bind to a variety of exogenous and endogenous ligands with different structures and physiochemical characteristics. ${ }^{7}$ Despite its reactivity to different ligands, the canonical AhR signaling pathway is tightly controlled. In particular, three different checkpoints regulate AhR activation: (a) proteasomal degradation of AhR, (b) metabolism of ligands by CYP1 A1, and (c) disruption of the AhR:Arnt complex by AhRR (Fig. 1a). ${ }^{18}$
\end{abstract}

Different studies have shown that several transcription start sites of AhR-responsive genes do not contain DRE, suggesting a non-canonical mechanism downstream of AhR. ${ }^{19-22}$ Indeed, after AhR ligand binding, AhR is able to directly interact with the hypophosphorylated retinoblastoma protein, leading to cell cycle arrest in the G1-phase in several cell lines, with a potential role in carcinogenesis control ${ }^{19,23}$ (Fig. 1b). A direct interaction of $A h R$ with nuclear factor- $\mathrm{KB}$ (NF-KB) was also described, inducing the expression of cytokines and chemokines, such as Bcell-activating factor of the tumor necrosis factor family, Blymphocyte chemoattractant, CC-chemokine ligand 1 (CCL1), and the transcription factor interferon responsive factor, ${ }^{24}$ and decreasing the expression of Cyp $1 A 1^{25}$ (Fig. 1C). AhR has also been shown to exhibit anti-estrogenic effects, notably by inducing degradation of the estrogen receptor through an E3 ubiquitin ligase-like activity ${ }^{26}$ (Fig. 1d). Finally, an interaction between AhR and the tumor suppressor Kruppel-like factor 6 (KLF6) at the non-consensus DRE has been characterized. ${ }^{27}$ KLF6 regulates the transcription of genes involved in different processes, including cellular proliferation, differentiation, and apoptosis, $^{28,29}$ and KLF6 mutations are linked to a number of cancers, such as hepatocellular carcinoma, ${ }^{30}$ gastric cancer, ${ }^{31}$ and colorectal cancer. ${ }^{32}$ Therefore, KLF6 is a tumor suppressor ${ }^{33}$ that can activate expression of $\mathrm{p} 21^{\mathrm{cip} 1}$ in an AhR-dependent manner, which leads to the inhibition of cell cycle progression ${ }^{34}$ (Fig. 1e). These data suggest that AhR and KLF6 are partners in anti-tumor signaling pathways.

Therefore, activation of AhR by a ligand can induce canonical and/or non-canonical pathways and subsequent transcriptional events. Additional studies are needed to determine the contribution of each of these signaling pathways to the effects of AhR agonists.

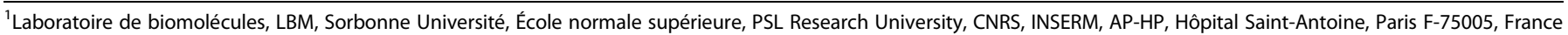
and ${ }^{2}$ Micalis Institute, Institut National de la Recherche Agronomique (INRA), AgroParisTech, Université Paris-Saclay, Jouy en Josas 78350, France Correspondence: Harry Sokol (harry.sokol@aphp.fr)
} 


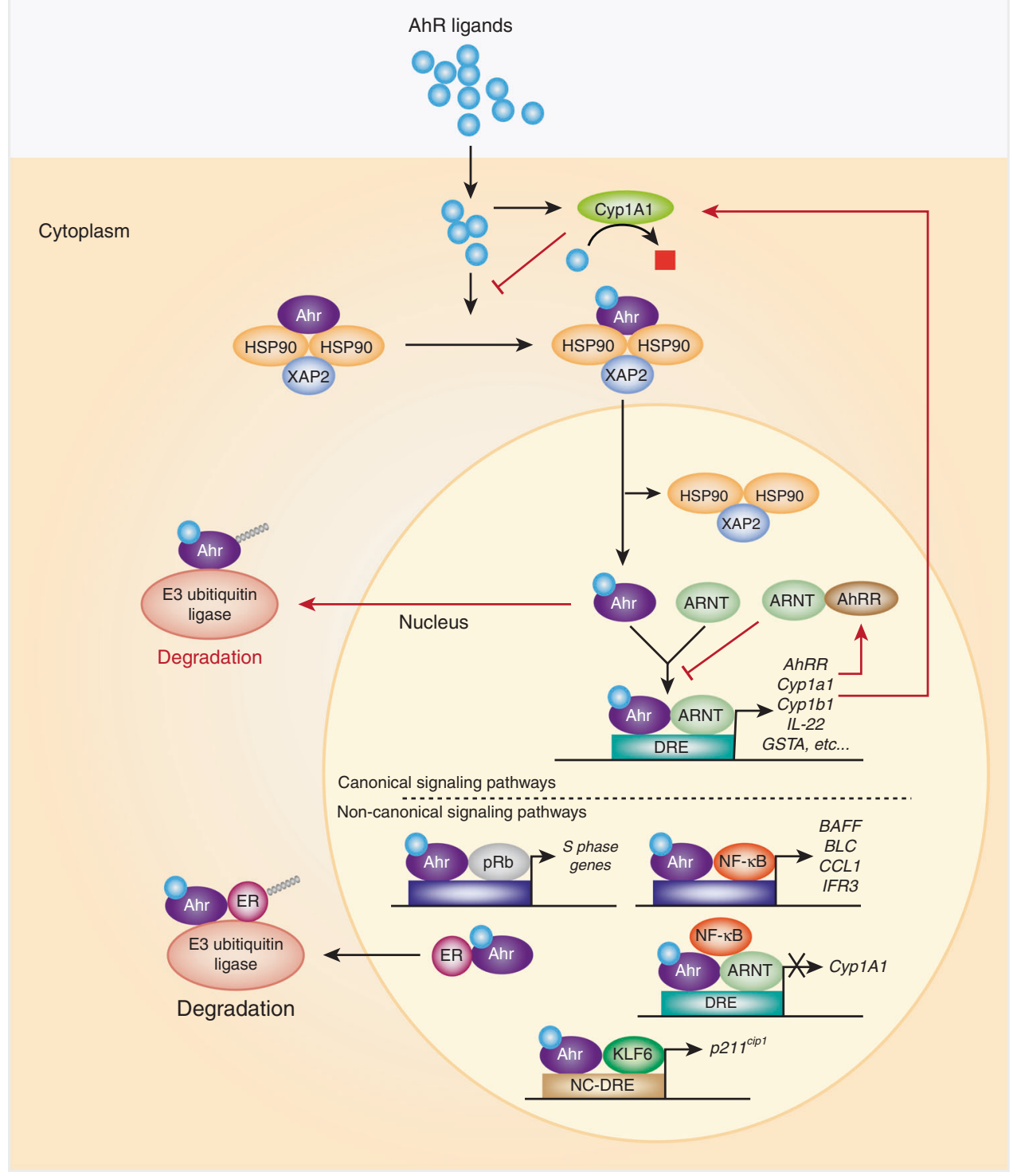

Fig. 1 Canonical and non-canonical signaling pathways of AhR. a Schematic representation of the canonical signaling pathways of AhR. In the absence of ligand, AhR is retained in the cytoplasm in an inactive complex containing chaperone proteins, such as HSP90 and XAP2. After ligand binding, AhR translocates into the nucleus, where it dimerizes with ARNT. The AhR/ARNT dimer binds genomic regions containing DRE that regulate expression of several phase I and phase II metabolizing enzymes, as well as several other genes, such as Cyp1a1, Cyp1b1, AhRR, II22, and GSTA. AhR activity is tightly controlled by three different negative feedback loops, including: (i) proteosomal degradation of AhR initiated by the ubiquitin ligase complex, (ii) metabolism clearance of ligands by CYP1A1, and (iii) disruption of the AhR/ARNT complex by AhRR. AhRR, AhR repressor; ARNT, AhR nuclear translocator; Cyp1a1, cytochrome P450 1A1; Cyp1b1, cytochrome P450 1B1; DRE, dioxin response element; GSTA, glutathione-S-transferase A; HSP90, heat shock protein 90; II-22, interleukin 22; XAP2, HBV X-associated protein 2. be Schematic representation of examples of non-canonical AhR signaling. $\mathbf{b}$ AhR activation by ligands may lead to a direct interaction with pRb, which blocks cell cycle progression by suppressing the expression of S-phase genes. pRb, hypophosphorylated retinoblastoma protein. $\mathbf{c}$ Through an interaction with NF- $\mathrm{KB}$, AhR induces the expression of cytokines and chemokines, such as BAFF, BLC, CCL1, and IFR3. The AhR/NF$\mathrm{KB}$ interaction also induces decreased expression of Cyp1A1. BAFF, B-cell-activating factor of the tumor necrosis factor family; BLC, Blymphocyte chemoattractant; CCL1, CC-chemokine ligand 1; IFR3, interferon responsive factor; NF- $\kappa B$, nuclear factor- $\kappa B$. d Ligand-activated AhR binds ER and promotes the proteolysis of ER by assembling a ubiquitin ligase complex. ER, estrogen receptor. e After ligand binding, AhR dimerizes with KLF6 and binds genomic regions containing NC-DRE that regulate the expression of target genes, such as p21 ${ }^{\text {cip } 1, \text { which }}$ mediate cell cycle control. KLF6, tumor suppressor Kruppel-like factor 6; NC-DRE, non-consensus DRE

\section{AHR LIGANDS}

Exogenous synthetic ligands

AhR ligands have varying levels of affinity and reactivity. To date, the majority of high-affinity AhR ligands are synthetic ligands, which are formed as a result of non-biological activity and include environmental contaminants, such as halogenated aromatic hydrocarbon $(\mathrm{HAH})$ and polycyclic aromatic hydrocarbon (PAH). ${ }^{7,35,36}$ Compared with PAHs, HAHs are metabolically stable and bind to AhR with higher affinity. Indeed, 2,3,7,8-tetrachlorodibenzo-p-dioxin (TCDD), a type of $\mathrm{HAH}$, is one of the wellcharacterized compounds that activates $A h R$, with as little as picomolar amounts, and is known to have detrimental toxic effects on the host. ${ }^{7,37,38}$ Other lower affinity synthetic AhR ligands, whose structure and chemical characteristics are different than those of $\mathrm{HAH}$ and $\mathrm{PAH}$ ligands, have been similarly identified; ${ }^{8}$ however, the effects of these ligands on mammalian 


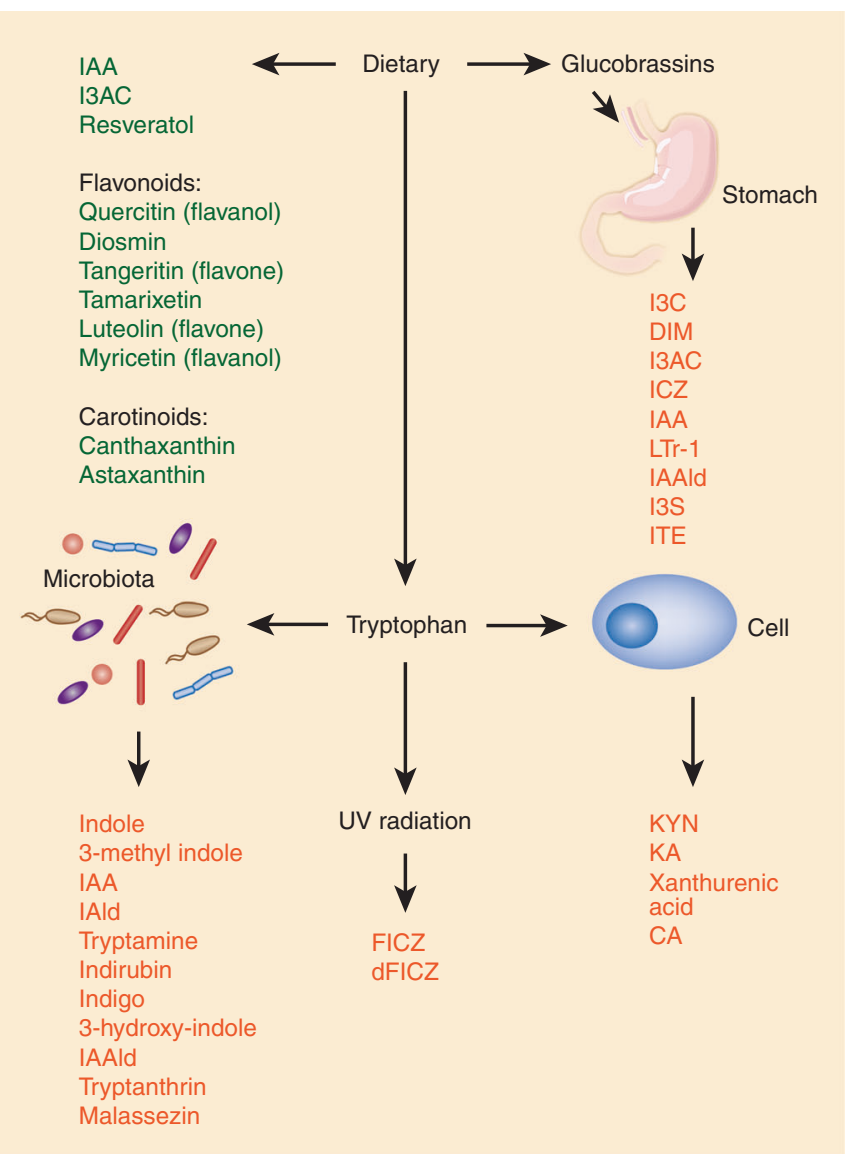

Fig. 2 Summary of endogenous and exogenous dietary-derived AhR ligands synthesis pathways. The diet is a major source of exogenous AhR ligands (green), as well as of tryptophan and glucobrassicins, which are endogenous AhR ligand precursors. Conversion of these compounds through biochemical reactions or the metabolic activity of microorganisms, such as the intestinal microbiota, leads to the production of endogenous AhR ligands (red). CA, Cinnabarinic acid; dFICZ, 6,12-diformylindolo[3,2-b]carbazole; DIM, 3,3'diindolylmethane; FICZ, 6-formylindolo[3,2-b]carbazole; IAA, Indole-3-acetic acid; IAld, Indole-3-aldehyde; IAAld, Indole3-acetaldehyde; ICZ, Indole[3,2-b]carbazole; I3S, Indoxyl-3-sulfate; ITE, 2-(1'H-indole-3' -carbonyl)-thiazole-4-carboxylic acid; I3AC, Indole-3-acetonitrile; I3C, Indole-3-carbinol; LTr-1, 2-(indol-3ylmethyl)-3,3'diindolylmethane; KA, Kynurenic acid; Kyn, Kynurenine

physiology are not yet clearly defined. Nonetheless, the identification of these synthetic AhR ligands emphasized the structural diversity of $A h R$ ligands and suggested the idea that their spectrum is likely to be much broader than was previously thought.

\section{Exogenous dietary-derived ligands}

Aside from synthetic ligands, a variety of naturally occurring ligands from dietary sources directly activate and/or inhibit the AhR signaling pathway (Fig. 2). As early as the 1970 s, several studies had already demonstrated that vegetable extracts or vegetable-derived materials induce CYP1A1 activity. ${ }^{39,40}$ Cruciferous vegetables, such as broccoli and brussel sprouts, have been found to be rich sources of glucobrassicin, which generates indole-3-carbinol (IBC) and indole -3-acetonitrile (IBAC) by enzymatic cleavage during mastication. Both IBC and IBACN are able to bind and activate AhR. ${ }^{40-43}$ Moreover, the ability of several dietary plant compounds, such as tryptophan, curcumin, and carotenoids, to bind AhR and/or induce AhR-dependent gene expression has also been reported. ${ }^{35,44-47}$

Other naturally occurring dietary AhR ligands are flavonoids (Fig. 2), which originate from plant-based foods, such as tea and fruits. Flavonoids are mostly AhR antagonists, but some of them, particularly quercetin, diosmin, tangeritin, and tamarixetin, are AhR agonists. ${ }^{47-52}$ One of the most interesting facts about these plant-derived AhR ligands is that they can be detected in human plasma in micromolar quantities, which are sufficient to inhibit/ activate AhR. ${ }^{53,54}$ Similar to synthetic compounds, these naturally occurring dietary-based AhR ligands have different structures, thus underscoring the heterogeneity of AhR ligands. It remains to be determined whether these dietary-derived AhR ligands have different downstream signaling effects and whether their different structure and physiochemical properties can be therapeutically exploited in different health care scenarios.

\section{Endogenous ligands}

The importance of AhR in normal development and host biochemical processes has been highlighted by studies using AhR knockout animals, which display numerous developmental and functional abnormalities. ${ }^{55,56}$ The lack of AhR activation by endogenous ligand(s) has been proposed to explain the functional alterations in AhR knockout mice; however, the identity of these putative compound/chemical(s) is not clear. ${ }^{7}$ Nonetheless, the ability of some of the presumptive endogenous ligands to activate AhR has been confirmed in vitro, ${ }^{7}$ but their specific role and physiological consequence in activating $A h R$ in vivo remains to be confirmed and/or identified.

\section{Indole and tryptophan metabolites}

Exogenous diet-derived indoles, such as tryptophan and glucobrassicin, are a major source of endogenous AhR ligand precursors. The conversion of these dietary-compound precursors into derivatives or byproducts through biochemical reactions or by metabolic activity of microorganisms, such as the intestinal microbiota, leads to the production of several important AhR ligands ${ }^{40,57}$ (Fig. 2).

In the presence of stomach gastric acid, glucobrassicin, I3C, and I3ACN are susceptible to non-enzymatic acid condensation reactions to generate a number of AhR ligands, including $3,3^{\prime}$ diindolylmethane (DIM), 2-(indol-3-ylmethyl)-3,3'diindolylmethane, and indolo[3,2-b]carbazole (ICZ) ${ }^{35,40,58-60}$ (Fig. 2).

Most indole derivatives are formed from the metabolism of tryptophan, which by itself is a form of indole, through various biological and physiochemical processes. In vitro and in vivo studies have shown that the byproducts of tryptophan photooxidation possess a high AhR-binding capacity and are able to induce the expression of Cyp1a1 and other AhR target genes. ${ }^{61-64}$ A prime example of a tryptophan photoproduct is a 6formylindolo[3,2-b]carbazole (FICZ), which displays a significant structural similarity and potency to ICZ and can agonistically stimulate AhR activity in as low as picomolar ranges ${ }^{65-67}$ (Fig. 2).

Aside from light exposure, some microorganisms can metabolize tryptophan into derivatives, such as indole acetic acid (IAA) and indole-3-acetaldehyde, both of which are AhR agonists ${ }^{68-72}$ (a list of known microorganisms that produce AhR ligands is found in Table 1) (Fig. 2). Some commensal bacteria, such as Escherichia coli, have up to three permeases for tryptophan transport and use tryptophan as a source of nitrogen. ${ }^{68}$ Moreover, indole is transported in and out of bacteria by passive diffusion or active transport by AcrEF-TolC and Mtr transporters. ${ }^{68,73,74}$ Another study showed that some strains of Lactobacillus reuteri and Lactobacillus johnsonii are able to produce indole-3-aldehyde (IAld) through the indole pyruvate pathway, which is catalyzed by the enzyme aromatic amino acid transferase. ${ }^{71}$ In mice, AhR activation by IAld administration promotes intestinal homeostasis by the induction of interleukin-22 (IL-22). ${ }^{71}$ L. reuteri is also able to shape gut 
Table 1. List of known microorganisms that produce AhR ligands

\begin{tabular}{|c|c|}
\hline Bacteria & AhR ligands \\
\hline Lactobacillus murinus & Indole derivative ${ }^{152}$ \\
\hline Bacillus alvei & Indole derivative ${ }^{194}$ \\
\hline Clostridium novyi & Indole derivative ${ }^{195}$ \\
\hline Clostridium limosum & Indole derivative ${ }^{196}$ \\
\hline Corynebacterium acnes & Indole derivative ${ }^{197}$ \\
\hline Enterococcus faecalis & Indole derivative ${ }^{198}$ \\
\hline Bacteroides thetaiotaomicron & Indole derivative ${ }^{199}$ \\
\hline Bacteroides sp. & Indole derivative ${ }^{200}$ \\
\hline Citrobacter $s p$. & Indole derivative ${ }^{201}$ \\
\hline Kleibsella planticola & Indole derivative ${ }^{206}$ \\
\hline Shigella flexneri & Indole derivative ${ }^{207}$ \\
\hline Vibrio cholera & Indole derivative 208 \\
\hline Lactobacillus bulgaricus OLL1181 & Not yet identified AhR ligands ${ }^{154}$ \\
\hline Kleibsella pneumonia & Indirubin, Indigo 209 \\
\hline Malassezia & Trypthantrin ${ }^{210}$ \\
\hline Propionibacterium freudenreichii ET-3 & 1,4-dihydroxy-2-naphtoic acid ${ }^{160}$ \\
\hline Malassezia & Malassezin ${ }^{210}$ \\
\hline Malassezia & Indirubin ${ }^{210}$ \\
\hline Providencia stuartii & Indirubin ${ }^{209}$ \\
\hline
\end{tabular}

intraepithelial lymphocytes (IELs) by metabolizing tryptophan in indole derivatives. ${ }^{72}$ Recent reports have demonstrated the importance of microbiota-derived indoles in regulating homeostasis through AhR activation, emphasizing the critical role of AhR in host physiology. ${ }^{75-77}$ Indigo and indirubin, both microbiota-derived tryptophan metabolites, have been isolated from human urine. A yeast-based AhR reporter assay showed that these tryptophan metabolites are almost equipotent as TCDD in their ability to activate AhR. ${ }^{78}$ However, the capacity of indigo and indirubin to activate AhR seemed to be much lower using a mammalian reporter system, highlighting the limitations posed by bioassays that measure AhR activity.

Tryptophan can also be metabolized by host cells via the enzymes indoleamine 2,3-dioxygenase and tryptophan 2,3-dioxygenase, which generate AhR ligands, such as kynurenine (Kyn) and kynurenic acid ${ }^{7,79-81}$ (Fig. 2). However, the Kyn concentration required to elicit reporter AhR activity in a hepatoma cell line casts doubt on its relevance as an AhR activator under physiological conditions. $^{78}$

Finally, serotonin and melatonin, two other tryptophan-derived biomolecules, are important neuroendocrine modulators, but their roles in activating AhR and the physiological consequence of this activation remain to be studied. Further work in this area may provide interesting insights into the AhR-dependent physiological effects of these tryptophan byproducts.

Other ligands

Interestingly, some chemicals, such as caffeine, ${ }^{82}$ nicotine $^{83}$ and pyridines, ${ }^{84}$ can induce AhR-dependent gene expression without binding to $A R^{85-90}$ (Table 2). These chemicals are not classical AhR ligands, but they may still have low-affinity binding capacity.
Indeed, carbaryl was previously reported to not bind $A \mathrm{hR}^{91,92}$ but modifications of the AhR ligand-binding assay demonstrated that it is actually a weak AhR ligand. ${ }^{37}$ As AhR activation affects a myriad of functions, it will be interesting to investigate the signaling pathways of these chemicals with ligand-independent AhR-activating properties to target specific AhR-related functions for health maintenance and disease prevention or treatment.

\section{EVOLUTIONARY CONSIDERATIONS}

AhR is evolutionary conserved across species. However, differential AhR activity and ligand reactivity exist across species, particularly between humans and murine animal models, which are often used for experimental purposes. ${ }^{93-95}$ For instance, mouse AhR has ten times higher affinity to TCDD compared with human AhR. ${ }^{96}$ In contrast, human AhR has higher affinity to indole derivatives compared with mouse AhR. ${ }^{96}$ The ability of human AhR to react less to environmental toxins, such as TCDD, compared with putative beneficial AhR ligands, such as indole, is reassuring for human health. However, further studies are needed to confirm the effects of different AhR ligands. Furthermore, AhR is known to interact with Hsps and the AhRHsp interaction has been implicated in cancer pathogenesis. ${ }^{97}$ AhR-Hsp interactions in humans, however, seem to be less stable than in mice, creating uncertainties in terms of the translational relevance of AhR-Hsp from murine findings. Human and murine AhR also share low transactivation domain sequence identity, ${ }^{98}$ highlighting further evidence of the discrepancy between humans and murine models. As most AhR studies rely on murine animal models, there is a need to confirm and complement these results with human data. 
Table 2. Chemicals with ligand-independent AhR-activating properties

Omeprazole

Thiabendazole

Lanosperole

Myristicin

Methylenedioxybenzenes

Caffeine

Insecticides (cypermethrin, diflubenyuron, tetrachlorvinphos)

Nicotine

Pyridines
Induce CYP1A1 expression ${ }^{85}$

Induce CYP1A1 expression ${ }^{86}$

Induce CYP1A1 expression ${ }^{87}$

Induce CYP1A1 expression ${ }^{88}$

Induce cytochrome $\mathrm{P} 450^{89}$

Induce CYP1A1/2 expression ${ }^{82}$

Induce CYP1A1/2 expression ${ }^{90}$

Induce CYP1A1 expression ${ }^{83}$

Induce CYP1A1/2 expression ${ }^{84}$
ROLE OF AHR IN EPITHELIAL AND IMMUNE INTESTINAL CELLS In the last decade, several seminal publications have highlighted the downstream effects of AhR signaling. Depending on the cellular context, AhR signaling influences several host responses and pathways, including cell cycle, neurological signaling, and immune responses, as well as responses to antioxidants, xenobiotics, and hormone-like estrogen. ${ }^{97,99-101}$

In the gastrointestinal tract, several reports have shown that AhR ligands modulate the development, function, production, and maintenance of several key mucosal immune cells and mediators. However, the exact molecular mechanisms are not well known. Moreover, depending on the context, the ligand, and the cell type involved, the effect of AhR modulation can vary.

Effect of AhR on intestinal epithelial cells and intestinal barrier function

The intestinal tract is the largest mucosal surface of the human body. It has a critical role in protecting the host from the environment, while maintaining proper nutrient absorption. The intestinal surface is lined by a single layer of intestinal epithelial cells (IECs), which forms a barrier between the intestinal lumen and the host's lamina propria (LP).

AhR influences different aspects of intestinal barrier function, from IEC proliferation to the production of IEC-derived molecules (Fig. 3). Proliferation of colonic crypt stem cells is impaired in $A h R^{-\prime}$ - mice, highlighting the fundamental role of $A h R$ in epithelial cell renewal and turnover. ${ }^{18}$

In terms of intestinal epithelial integrity, the AhR agonist FICZ had been shown to prevent hypoxia-driven barrier dysfunction of IEC in vitro. ${ }^{102}$ In particular, AhR activation ameliorated the hypoxia-driven intestinal permeability by rectifying expression of the epithelial tight junction protein ZO- $1 .{ }^{102}$ Similarly, bacterialderived indole was able to increase epithelial tight junction integrity and reduce inflammation, ${ }^{75}$ although it is still unclear whether this effect is AhR-mediated. Moreover, mice with intestinal epithelium-specific AhR deficiency were more sensitive than control mice to Dextran Sodium Sulfate (DSS)-induced intestinal inflammation and had enhanced apoptosis of epithelial cells, suggesting that AhR has an intrinsic role in IEC integrity. ${ }^{103}$

Although it is significant to elucidate the direct impact of $A h R$ on IEC, it is equally important to remember that AhR is expressed by other cell types, including immune cells (Fig. 3). Consequently, the effect of AhR on IEC may also be indirectly mediated through signaling with other cell types. The most obvious example is the AhR-dependent production of IL-22 by immune cells that have major effects on IEC, inducing their proliferation and the production of antimicrobial peptides ${ }^{104,105}$ (Fig. 3). Conversely, IEC may modulate the effects of AhR ligand on other cell types. Indeed, CYP1A1 deficiency in IEC has been shown to increase the availability of AhR ligands to immune cells, suggesting that IEC have a crucial role as gatekeepers for AhR ligand availability in the intestine. ${ }^{106}$ Thus, reductionist in vitro studies that only involve IEC may not be sufficient to recapitulate the global effect of AhR on IEC. Studies using hematopoietic chimeras and conditional AhR knockout mice could be useful to determine the in vivo effect of AhR on different cell types and, notably, to discriminate the direct and indirect cellular effects of AhR signaling. Certainly, studies using murine models with conditional AhR gene deletion in dendritic cells (DCs) or IECs have demonstrated that AhR signaling in these cell types is dispensable for the normal development of intestinal lymphoid organs, such as cryptopatches and isolated lymphoid follicles, ${ }^{107}$ but has an effect on other cell types, such as T cells.

Aside from their global effects on epithelial cells, AhR ligands influence the gene expression and protein production of specialized IEC types. ${ }^{55}$ For instance, microbiota-derived indole induces the production of incretin and hormones by enteroendocrine cells. ${ }^{108}$ Whether this is an AhR dependent response is not yet known, but it suggests the possible involvement of AhRrelated signaling in hormonal function of the epithelium.

AhR is involved in mucus production in the lung, suggesting that it may be similarly involved in mucin production in the intestine. ${ }^{109}$ However, no study at this time has demonstrated the ability of AhR to regulate mucus production in the intestine.

Effect of AhR on intestinal immune cells As AhR is expressed by different intestinal immune cells, such as IELs, ${ }^{110}$ Th17 cells, ${ }^{111}$ innate lymphoid cells (ILCs), ${ }^{112}$ macrophages, $^{3,113} \mathrm{DCs},{ }^{3,114,115}$ and neutrophils ${ }^{116,117}$ (Fig. 3), AhR signaling is pivotal to the regulation of mucosal intestinal immune responses.

Intraepithelial lymphocytes

Specialized IELs, such as TCR $\gamma \delta$ and TCRa $\beta$ CD8aa T cells, are strategically positioned between IECs. Along with IECs, IELs represent a critical first line of defense, as they are involved in immune surveillance and in modulating intestinal inflammatory responses. ${ }^{18,110}$ AhR deficiency in mice results in the loss of intestinal $\gamma \delta T$ cells and TCRaß CD8aa IELs in a cell-intrinsic manner, possibly through a reduced survival mechanism; ${ }^{110}$ this suggests the necessity of AhR signaling for postnatal IEL survival. As IELs are also involved in epithelial growth, ${ }^{18}$ it follows that AhRdependent IEL maintenance contributes to IEC homeostasis and further suggests the indirect ability of AhR to regulate intestinal barrier function (Fig. 3).

Innate lymphoid cells. ILCs represent a unique intestinal immune cell type that populates the intestinal LP. ILCs participate in the maintenance of intestinal homeostasis and protection against infection. Similar to IELs, AhR is a critical modulator of ILCs. ${ }^{110,118}$ 


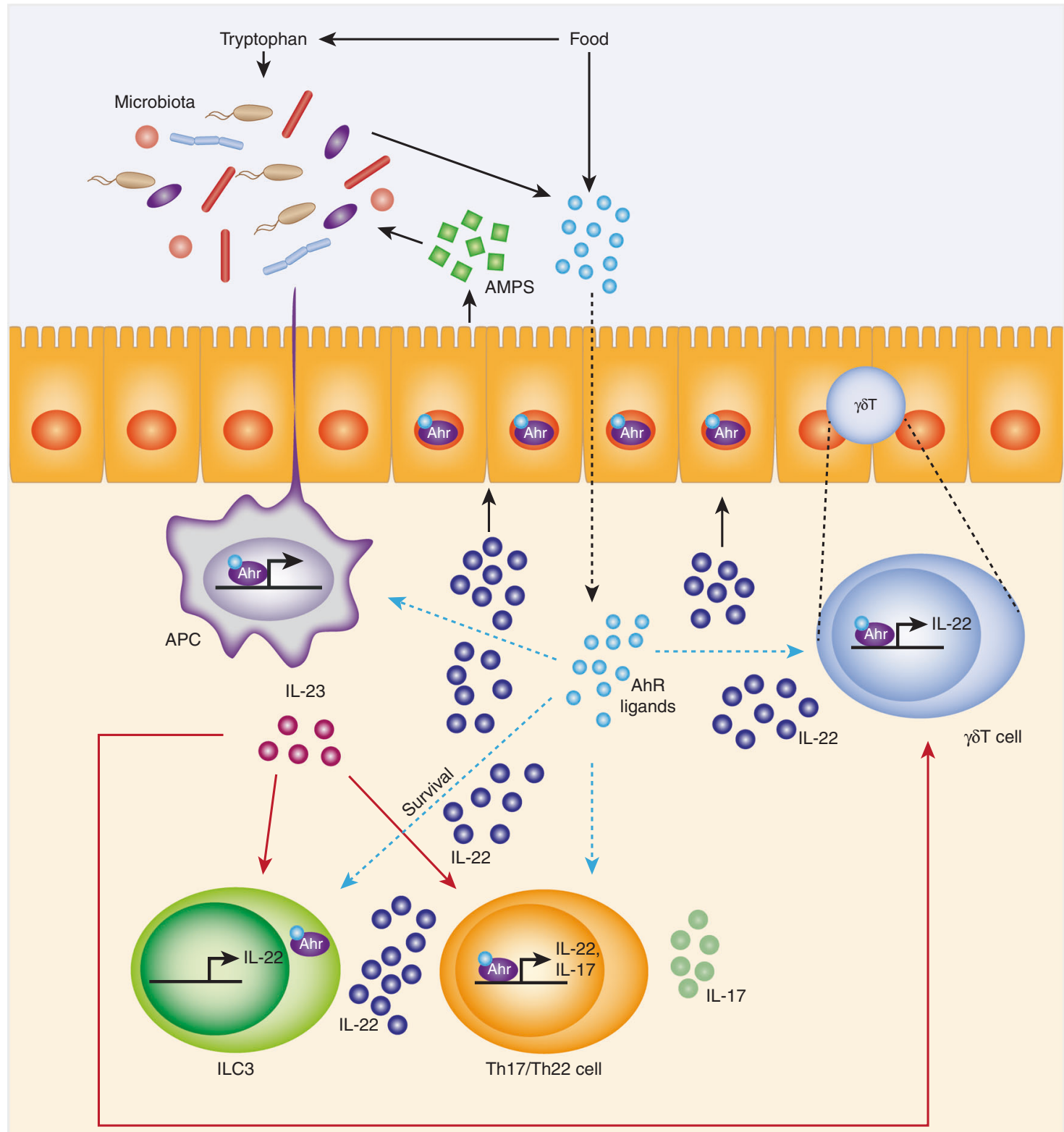

Fig. 3 Physiological effects of AhR in the gastro-intestinal tract. AhR ligands can be generated from the diet and/or microbiota and are able to activate AhR. In the intestine, AhR is expressed by intestinal epithelial cells (IEC), where it is involved in epithelial cell renewal and turn over. IECs have a role in the regulation of AhR ligand availability to intestinal immune cells, including antigen presenting cells (APCs), innate lymphoid cells (ILCs), Th17/Th22 cells, and intraepithelial lymphocyte $\gamma \delta$ T cells ( $\gamma \delta \mathrm{T}$ cells). All of these immune cells express AhR and its signaling is pivotal in the regulation of mucosal intestinal immune responses. Indeed, AhR is essential for the development and function of APCs. IL-23 is produced by APC in an AhR-independent manner but its effect on IL-22 production by ILC, Th17/Th 22 and $\gamma \delta$ T is mediated by AhR and RORyt. IL-22 is involved in mucosal wound-healing and it mediates innate antimicrobial resistance by inducing production of antimicrobial peptides (AMPs) by intestinal IECs. AhR is important for the development, maintenance, and function of ILC and $\gamma \delta$ T cells, which have immune defense function. AhR is also essential for the function of Th22 and Th17 cells. Beyond its inflammatory pathology connotation, Th17 cells are important drivers of health, especially as they provide barrier protection and are involved in pathogen defense

In particular, both the production and function of a specific subgroup of ILCs, termed Group 3 ILC (ILC3), which includes lymphoid tissue inducer (LTi), natural cytotoxicity triggering receptor (NCR) + ILC3, and NCR-ILC3, are highly influenced by AhR activation ${ }^{110,118}$ (Fig. 3). AhR deficiency in the hematopoietic compartment leads to profound loss of LTi and NCR + ILC3 in the intestinal LP and reduced IL-22 production. ${ }^{10,118,119}$ ILC3 produce the cytokines IL-17 and/or IL-22 through the transcription factor RORyt, and the lack of ILC-derived cytokines, particularly IL-22, has been linked to different host pathologies, including infections and metabolic disorders. ${ }^{119}$ In addition, IL-22, which is also produced by $T$ cells (see below), is implicated in intestinal homeostasis, ${ }^{71,120}$ notably by inducing the expression and production of antimicrobial peptides, such as Reg3 $\gamma$ and Reg $3 \beta^{104,105}$ by IEC (Fig. 3). ILCs are also involved in the postnatal development of intestinal lymphoid follicles, including cryptopatches and isolated lymphoid follicles.

Myeloid cells. AhR is highly expressed in different innate immune cells, particularly antigen presenting cells (APCs) and neutrophils. APCs form a bridge between the innate and adaptive immune systems. Mucosal APCs are involved in inflammatory and tolerance 
responses by driving either Th17 or regulatory T (Treg) cells. ${ }^{121,122}$ These functions are partly modulated by the local environment, such as the presence of AhR ligands. ${ }^{122,123}$ In response to lipopolysaccharide (LPS), macrophages derived from $A h R^{-1-}$ mice showed an increase production of pro-inflammatory cytokines, ${ }^{113}$ whereas the production of IL-10 by $A h R^{-/-}$bone marrow-derived DCs (BMDCs) was decreased. ${ }^{114}$ Moreover, the absence of AhR in BMDCs inhibits Treg development, facilitates Th17 generation from naive $T$ cells and promotes naive T-cell proliferation in vitro. ${ }^{114}$ AhR has also recently been identified as a molecular switch for monocyte fate, promoting differentiation toward DC while impairing differentiation into macrophages. ${ }^{124}$ Combined, these studies suggest the ability of AhR to regulate the antiinflammatory responses of APCs. However, intestinal APCs are very specialized and are different from bone marrow-derived APCs used in these studies. Therefore, AhR could have different effects in intestinal APC subpopulations. An accumulation of plasmacytoid DCs was observed in lymph nodes of $A h R^{-/-}$mice compared with wild-type (WT) mice. ${ }^{123}$ Another study showed that the percentage of peritoneal monocyte-derived DCs was decreased after antibiotic treatment, which induced the loss of intestinal bacteria producing AhR ligands. ${ }^{124}$ Moreover, an I3Csupplemented diet rescued monocyte-derived DC differentiation in antibiotic-treated mice. ${ }^{124}$ These results suggest that AhR is a modulator of intestinal DCs, with potential indirect impacts on intestinal T cell responses (Fig. 3). Intestinal DCs and macrophages are also involved in the AhR-mediated maintenance of intestinal epithelium integrity. Indeed, AhR deletion in CD11c-expressing intestinal mucosal DCs and certain macrophage subsets led to increased numbers of epithelial stem cells and goblet cells but decreased numbers of Paneth cells in the small intestine. ${ }^{125}$ Moreover, as intestinal DCs and macrophages produce IL-23, these cells participate also to AhR-mediated maintenance of intestinal homeostasis and protection against infection. Indeed, after contact with microbial product, intestinal DCs and macrophages produce, independently of AhR, the cytokine IL-23, which mediates, in association with AhR signaling, the production of IL-22 by ILCs and T cells ${ }^{118,126-130}$ (Fig. 3).

Finally, AhR is also involved in neutrophil functions, particularly cytokine production. IL-17 and IL-22 production by neutrophils is also mediated by IL-23 through AhR and RORyt. ${ }^{17}$ This is of particular importance in the context of intestinal homeostasis, because the protective effects of neutrophils in colitis are at least partly mediated by their production of IL-22. ${ }^{116,117}$ Comprehensively, these studies highlight the critical role of AhR in orchestrating innate immune responses and homeostasis in the intestine.

$T$ cells. In addition to innate immune responses, AhR is involved in coordinating adaptive mucosal immune responses and homeostasis. AhR is highly expressed in Th17/22 cells, weakly expressed in Foxp $3+$ Treg cells and almost undetected in Th1 or Th2 cells. ${ }^{111,131}$ Similar to ILCs, AhR is also essential for IL-22 production by $T$ cells. In vitro studies showed that AhR promotes Th17 cell differentiation and the production of IL-17 and IL-22 by $\gamma \delta \mathrm{T}$ cells. ${ }^{132,133}$ In contrast, AhR expression is reduced in pathogenic Th17 cells, which promotes tissue inflammation, ${ }^{134}$ and T cells from $A h R^{-/-}$mice develop Th17 responses but lack the ability to produce IL-22, ${ }^{131}$ suggesting the capacity of AhR to modulate inflammatory responses. It is important to remember that, beyond its inflammatory pathology connotation, Th17 cells are important drivers of health, especially as they provide barrier protection and are involved in pathogen defense. ${ }^{111}$ As reviewed below, the AhR-mediated Th17 response is one key factor in immune defense against infections. It is important to note that intestinal $T$ cells are specialized and different from $T$ cells generated in vitro or isolated from the spleen, which are used in the majority of studies exploring the role of AhR in T cells.
The deletion of AhR in T cells attenuated colitis by suppressing Th17 cell infiltration into the LP. ${ }^{103}$ Moreover, differences in the frequencies of intestinal effector $T$ cell subsets were observed in $A h R^{-/-}$mice compared with WT mice after DSS treatment. ${ }^{135}$ The percentage of Th17, Th1, and Tc1 (type1 CD8 \pm T cells) but not Tc17 (IL-17 \pm CD8 $\pm T$ cells) was increased in the colonic LP of $A h R^{-/-}$mice compared with WT mice. ${ }^{135}$ In the small intestine, elevated frequencies of Th17 and Tc1 were also observed in $A h R^{-/-}$ mice. ${ }^{135}$ These results show that the AhR pathway has an intrinsic role in T-cell functions, particularly in the intestinal mucosa (Fig. 3).

In the last decade, several mechanisms of actions by which AhR promotes the expression of IL-17 in Th17 cells have been proposed. ${ }^{132,133,136}$ Regarding the role of $A h R$ in the induction of IL-22 production by T cells, an interaction with the transcription factor RORyt has been identified. Indeed, chromatin immunoprecipitation assay showed that AhR is recruited to IL-22 locus when HEK293T cells coexpress RORyt ${ }^{118}$. A study using thymoma cell lines provided clues that cooperation between AhR and RORyt may be involved in the induction of IL-22 expression. ${ }^{18}$ IL-22 expression by $\mathrm{T}$ cells might also be regulated other cytokines. Indeed, effects of IL- 6 and IL-21 on Th22 cells generation seems to be AhR-independent and AhR-dependent respectively. ${ }^{137,138}$

AhR also participates in intestinal homeostasis by regulating the generation and function of Treg cells. ${ }^{139}$ Consistent with the importance of AhR in gut tissue-associated Treg cells, ${ }^{139}$ high amounts of AhR have been observed in Treg cells from the gut compared to Tregs from other anatomical locations. ${ }^{140}$ Expression of the transcription factor Foxp3, which drives Treg differentiation and function, is decreased in $A h R^{-/-}$mice. ${ }^{141}$ Moreover, AhR activation by agonists induced Foxp3 expression and promoted Treg differentiation. ${ }^{139,142,143}$ In contrast, no increase in Treg differentiation or function has been observed in mice with constitutive AhR activation, ${ }^{144}$ suggesting a complex regulatory system. Thus, the exact role of AhR in Treg differentiation and function remains controversial and warrants further studies.

The type 1 Treg cells (Tr1) subset also expresses AhR. Tr1 cells express IL-10 but not Foxp3 and are prominent in chronic infections and other specific immune-related manipulations, such as peptide immunization or anti-CD3 activation. ${ }^{145} \mathrm{IL}-10$ and IL-21 production by $\operatorname{Tr} 1$ is controlled by $A h R$ via interaction with the transcription factor c-Maf. ${ }^{146}$ Interestingly, AhR activation, in the presence of TGF- $\beta$, can promote the differentiation of Th17 cells into $\operatorname{Tr} 1$ cells, $^{147}$ underscoring the role of AhR in T-cell plasticity.

Collectively, these data demonstrate that AhR has an important role in intestinal homeostasis by directly and indirectly modulating the function of epithelial cells and many immune cell subtypes. Although AhR activation appears to be essential at basal homeostatic state, its protective role is more clearly unmasked in altered settings such as intestinal inflammation or infection.

\section{ROLE OF AHR IN INTESTINAL PATHOLOGICAL CONTEXT}

AhR signaling regulates the composition and function of different cell types in the gastrointestinal tract and has a pivotal role in maintaining the balance between health and disease. Because of its numerous functions, AhR has been implicated in several intestinal pathologies. The following section highlights the role of AhR in intestinal inflammation, infection and cancer.

\section{AhR in gut inflammation}

Accumulating evidence has shown that AhR has an important role in the regulation of the immune response to Crohn's disease (CD) and ulcerative colitis (UC), which are the two main subtypes of inflammatory bowel disease (IBD). The pathogenesis of IBD is unclear but involves an exaggerated intestinal immune response toward the gut microbiota in genetically susceptible hosts.

AhR gene and protein expressions are lower in inflamed tissue from CD patients, but not UC patients, compared with uninflamed 


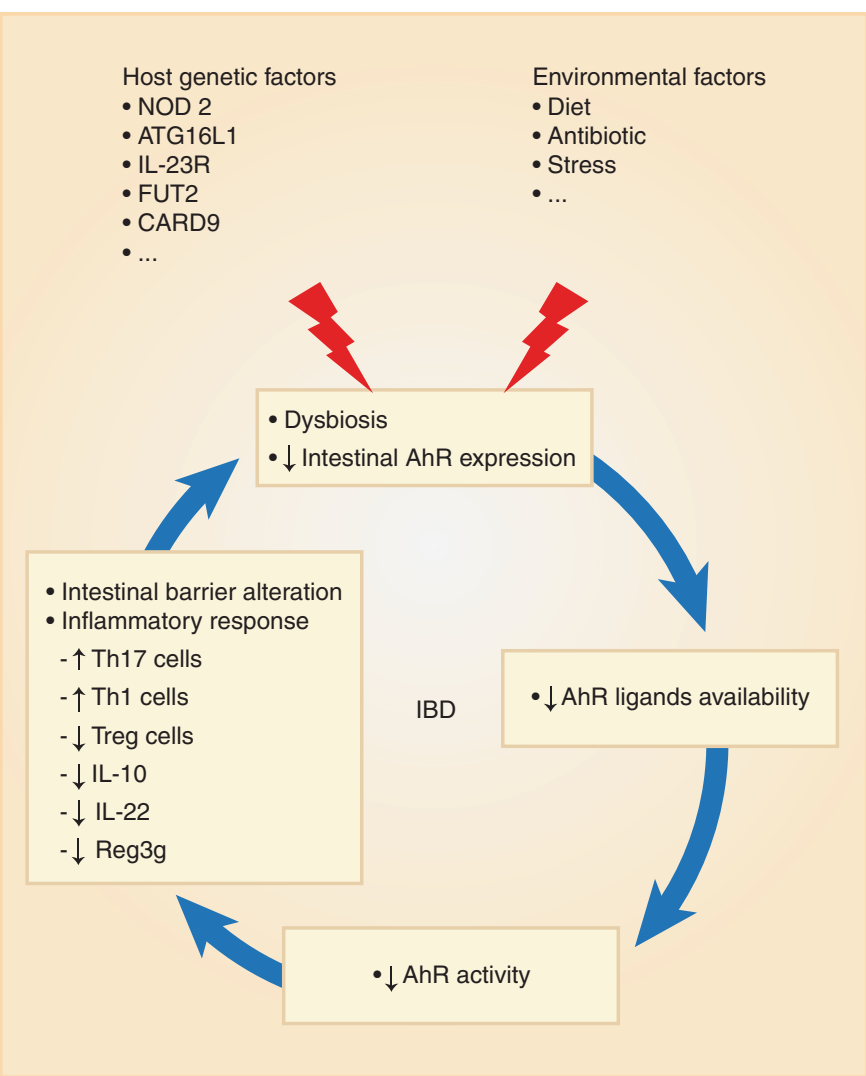

Fig. 4 Potential role of $A h R$ in inflammatory bowel disease. In inflammatory bowel disease (IBD), a combination of genetic factors and environmental factors leads to decreased intestinal AhR expression and dysbiosis, which in turn reduces the production of AhR ligands by the microbiota. This decreased availability of AhR ligands in the intestine leads to defective AhR activation, which contributes to intestinal barrier and immune response alterations, and amplifies the dysbiosis in a vicious cycle favoring chronic inflammation and consequently IBD

tissues and healthy controls ${ }^{148}$ (Fig. 4). However, other studies have shown that, unlike AhR protein expression, which is decreased in inflamed CD tissues, there is no difference in AhR gene expression between tissues from $C D$ patients and healthy controls. ${ }^{149}$ The involvement of post-transcriptional mechanisms may explain the disparity between $A h R$ gene and protein expression. This is consistent with a study showing that miRNA124 , which is involved in post-ranscriptional gene regulation and had been shown to have a pro-inflammatory role in TNBS-induced colitis by suppressing $A h R$, is highly expressed in inflamed biopsies and IECs from CD patients. ${ }^{150}$

The importance of AhR in intestinal homeostasis is underscored by murine studies showing that AhR deficiency increases the immunopathology of DSS- and T-cell transfer-induced colitis. ${ }^{110,112}$ Interestingly, immune cells from IBD patients, particularly CD patients, tend to express low levels of AhR. For instance, flow cytometry analysis of the LP mononuclear cells demonstrated that the expression of $\mathrm{AhR}$ in $\mathrm{CD}^{+}, \mathrm{CD}^{+}, \mathrm{CD}^{+} 6^{+}$, and $\mathrm{CD} 25^{+}$cells is decreased. ${ }^{148}$ Similarly, the expression of AhR in NKp44 ${ }^{+}$ILC3s cells from the inflamed intestinal biopsies of $C D$ patients was lower compared to ILC3 from unaffected tissues. ${ }^{151}$ The cause for this downregulation is unknown. However, as AhR ligands, especially those that are microbiota derived, have been shown to be impaired in IBD patients, ${ }^{152}$ it is tempting to hypothesize that decreased AhR expression in immune cells is related to decreased ligand availability (Fig. 4).
Administration of AhR agonists and antagonists in different animal models of colitis has also been utilized to elucidate the role of AhR in gut inflammation. After FICZ treatment, the severity of Trinitrobenzenesulfonic acid (TNBS)-, DSS-, and T-cell-transferinduced colitis in mice significantly decreased, which was characterized by down-regulation of pro-inflammatory cytokines and production of IL-22. ${ }^{148,152}$ In contrast, treatment of mice with an AhR antagonist reduced IL-22 production and enhanced the severity of inflammation in a murine model of TNBS-induced colitis. Moreover, administration of anti-IL-22 prevented the antiinflammatory effect of FICZ, suggesting that the therapeutic effect of FICZ, at least in this colitis model, is partially mediated by IL$22 .{ }^{148}$ Moreover, oral administration of the AhR agonist $\beta$ naphthoflavone decreased DSS-induced colitis severity and the production of pro-inflammatory cytokines, such as tumor necrosis factor (TNF)- $a$, IL- 6 , and IL- $1 \beta .{ }^{153}$ The severity of DSS-induced colitis was also attenuated by the toxic synthetic AhR ligand TCDD, presumably through up-regulation of colonic PGE2 production. ${ }^{154}$ The TCDD effects were further attributed to increased differentiation of Tregs and decreased induction of Th17 cells through epigenetic regulation. ${ }^{155}$ Activation of AhR using the non-toxic agonist 2-(1'H-indole-3'-carbonyl)-thiazole-4-carboxylic acid methyl ester (ITE) similarly induced human Tregs in vitro. ${ }^{142}$ Moreover, in a humanized mice model, where human $\mathrm{CD}^{+}{ }^{+} \mathrm{T}$ cells drive TNBS-induced colitis, ITE administration ameliorated colitis severity by upregulating CD39-expressing Granzyme B and IL-10secreting human Tregs. ${ }^{142}$ The effect of AhR ligands in reversing inflammatory responses has also been demonstrated in a clinical setting. In fact, treatment of LP mononuclear cells from CD patients with the AhR agonist FICZ resulted in decreased IFN- $\gamma$ expression and up-regulation of IL-22. ${ }^{148}$ Overall, these studies highlight the important role of AhR signaling in modulating intestinal inflammation (Fig. 4).

As indicated above, some AhR ligands are byproducts of tryptophan metabolism by the gut microbiota; these derivatives have been shown to modulate the intestinal immune response. Recently, our group demonstrated that dysbiotic microbiota from mice deficient for Caspase recruitment domain 9 (CARD9), one of the numerous IBD susceptibility genes, fails to metabolize tryptophan, leading to defective production of AhR agonists. ${ }^{152}$ This defect led to impaired IL-22 production and ultimately contributed to the increased susceptibility of $\mathrm{Card9}^{-/-}$mice to DSS-induced colitis. ${ }^{152}$ FICZ administration was effective in rescuing the IL-22 defect and subsequently decreasing the

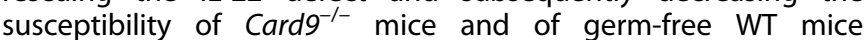
colonized with $\mathrm{Card}^{-/-}$microbiota to colitis. ${ }^{152}$ Similar to what was observed in $\mathrm{Card9}^{-} \digamma^{-}$mice, feces of patients with IBD, particularly in those with CARD9 risk alleles, showed impaired AhR agonist activity, consistent with decreased concentrations of the indole derivative IAA. ${ }^{152}$ No association was observed among other major IBD-associated loci, including NOD2, ATG16L1, and $\angle R R K 2{ }^{152}$ suggesting a specific connection between IBD, CARD9, and the ability of the microbiota to produce AhR agonists. Likewise, the AhR-IL-22 axis shapes the microbiota to produce AhR ligands and consequently is involved in the maintenance of gut immune homeostasis. Administration of exogenous IL-22 was sufficient to normalize AhR ligand production and colitis susceptibility in $\mathrm{Card9}^{-/-}$mice, suggesting a vicious circle between the AhR ligand defect and IL-22 defect. ${ }^{152}$ Abundance of segmented filamentous bacteria (SFB), a major driver of Th17 cell development in the intestine, ${ }^{156,157}$ is regulated by IL17 signaling in epithelial cells. ${ }^{158} \mathrm{IL}-22$ production by $\mathrm{AhR}^{+} \mathrm{RORyt}^{+} \mathrm{ILC}$ has also been shown to be crucial to control SFB colonization and the Th17 cell response. ${ }^{112}$ Decrease IL-22 production due to the loss of ILC3 in $A h R^{-1-}$ mice was associated with SFB outgrowth and enhanced Th17 responses, ${ }^{112}$ suggesting that AhR-dependent ILC3 survival, and consequently its IL-22 production, has an important role in shaping the gut microbiota and in intestinal homeostasis (Fig. 4). 


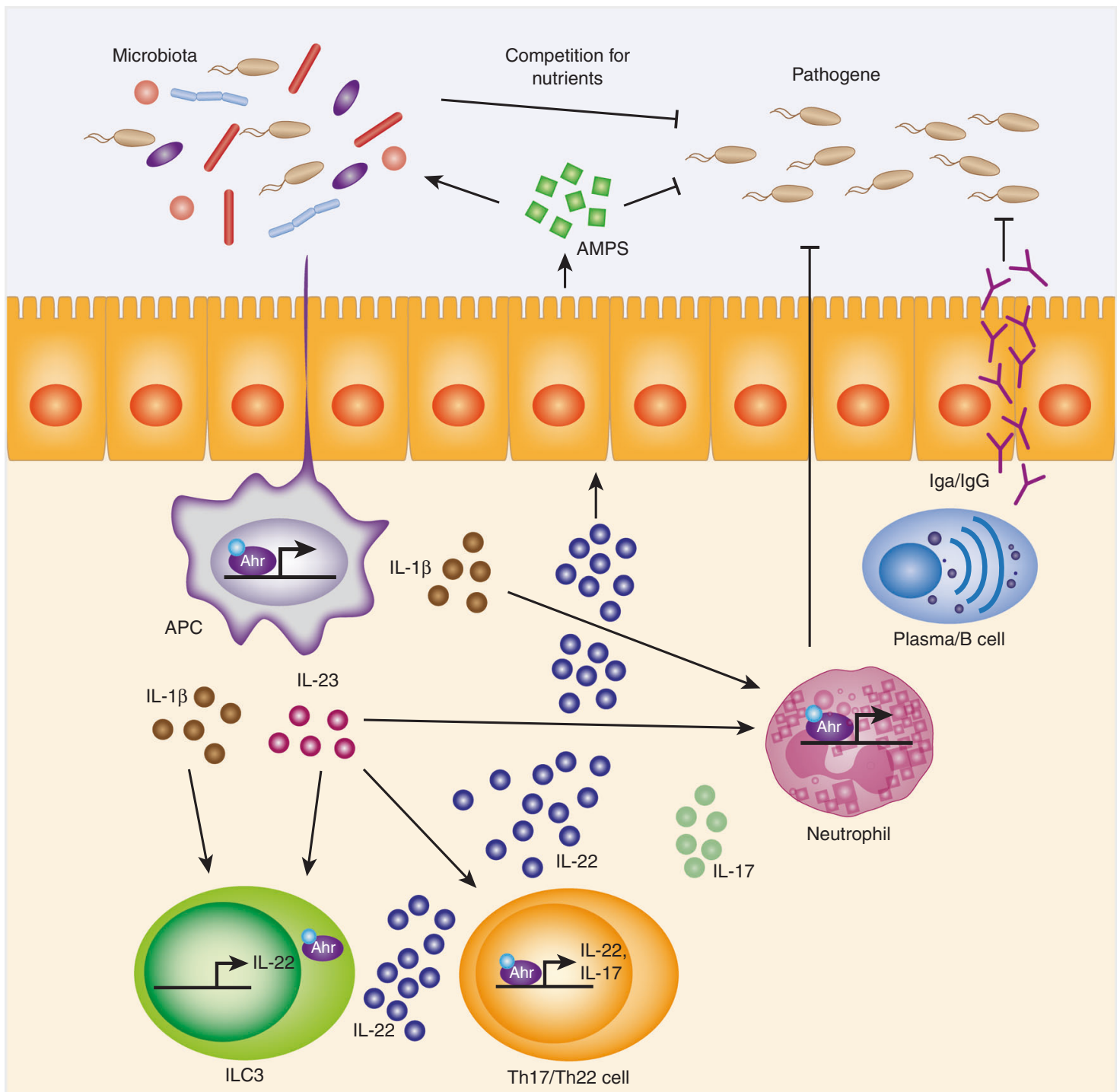

Fig. 5 Effects of AhR on colonization resistance against intestinal pathogens. Several immune mechanisms work in concert with the intestinal microbiota and contribute to colonization resistance against intestinal pathogens. AhR activation enhances the production of IL-1 $\beta$ by antigen presenting cells (APCs), which promotes neutrophil recruitment and pathogen eradication. AhR, in association with IL-23, is essential for the function of innate lymphoid cells (ILCs) and Th17/Th22 cells that control both commensals and pathogens through IL-22 production, inducing the production of antimicrobial peptides (AMPs) by intestinal epithelial cells. AMPs are also involved in colonization resistance against pathogens by shaping the microbiota that is then able to compete with pathogens for nutrients and regulate the secretion of intestinal IgA and IgG by $B$ cells, contributing to pathogen eradication

Certain bacteria and fungi can metabolize tryptophan into indole derivatives (Table 1), $i^{71,152,159,160}$ henceforth, these microorganisms are attractive candidates to be used as next generation probiotics to reduce intestinal inflammation. In fact, a recent study from our group demonstrated that administration of three Lactobacillus strains (L. murinus, L. reuteri, and L. taiwanensis) isolated from the feces of WT mice for their ability to activate AhR was efficient in rescuing the impaired IL-22 production and susceptibility to DSS of germ-free WT mice colonized with $\mathrm{Card}^{-/-}$ microbiota. ${ }^{152}$ These effects were mediated by AhR, as they were abrogated in the presence of an AhR antagonist. ${ }^{152}$ Moreover, Propionibacterium freudenreichii ET-3, isolated from Swiss-type cheese, has been shown to produce the AhR agonist 1,4dihydroxy-2-naphthoic acid, which can induce expression of the intestinal anti-microbial peptides Reg $3 \gamma$ and Reg $3 \beta$ and can improve DSS-induced colitis in mice. ${ }^{160}$ Lactobacillus bulgaricus OLL1181 is also able to activate the AhR pathway and improve
DSS-induced colitis. ${ }^{154}$ Overall, these studies emphasize the importance of microbiota-derived AhR ligands in modulating intestinal inflammation (Fig. 4).

In recent years, specific lifestyle choices or diet have been proposed as contributing factors in the rising inflammatory diseases in developed countries. As detailed above, AhR activators can also be derived from diet. Hence, it is possible that insufficient sources of AhR ligands may underlie how certain diets can influence the evolution of inflammatory diseases (Fig. 4). As diet can modulate intestinal microbiota composition, ${ }^{161,162}$ it may contribute to inflammatory disease by changing the capacity of the microbiota to produce AhR ligands. Accordingly, a tryptophanfree diet altered gut microbiota and increased the susceptibility of animal models to DSS- and TNBS-induced colitis. ${ }^{163}$ On the other hand, a tryptophan-containing diet decreased DSS-induced colitis severity ${ }^{164,165}$ and regulated epithelial homeostasis through AhR activation. ${ }^{165}$ Henceforth, activation of the AhR pathway by 
dietary-derived tryptophan derivatives, such as indoles, and/or probiotics organisms that produce AhR ligands offers exciting promise for future therapies in IBD and other diseases where individuals have dysbiotic microbiota with defective production of AhR agonists.

\section{AhR and intestinal infection}

In association with innate and adaptive immunity, the microbiota controls resistance against colonization by intestinal pathogens. AhR ligand activation induces key immune responses, notably IL22 production, that are critical for intestinal pathogen clearance (Fig. 5). Indeed, AhR deficiency in mice results in higher susceptibility to Citrobacter rodentium, ${ }^{106,107,112,126}$ which is a natural mouse pathogen widely used to mimic human infections caused by enteropathogenic $E$. coli and enterohemorrhagic $E$. coli. ${ }^{166}$ AhR regulates host immunity to $C$. rodentium in an IL-22dependent manner, as $A h R^{-/-}$mice fail to produce IL$22,106,107,112,126$ and ectopic expression of IL-22 protects $A h R^{-/}$ mice from this pathogen. ${ }^{112}$ Moreover, AhR and IL-23R-mediated IL-22 production of ILC3 are essential for shaping the microbiota to mediate early colonization resistance against $C$. rodentium infection $^{167}$ (Fig. 5). Higher susceptibility to $C$. rodentium is similarly observed in $R 26^{\text {Cyp } 1 a 1}$ mice, where constitutive Cyp1a1 expression depletes the reservoir of natural AhR ligands and generates a quasi AhR-deficient state. ${ }^{106}$ Administration of recombinant IL-22 or an I3C-supplemented diet reduced bacterial burden and prolonged the survival of $R 26^{\text {Cyp } 1 a 1}$ mice. ${ }^{106}$ Conversely, deletion of the three AhR-controlled CYP enzymes CYP1A1, CYP1A2, and CYP1B1 had the opposite effect. ${ }^{106}$

$A h R^{-1-}$ mice are hypersensitive to LPS-induced septic shock, suggesting that AhR may also be involved in immune responses toward other Gram-negative bacteria. ${ }^{116,168-170}$ Specifically, upon LPS treatment, the serum levels of IL-6, IL-1 $\beta$, and TNF- $\alpha$ in $A h R^{-/-}$ mice increased, whereas the level of IL-10 decreased compared with WT controls. ${ }^{116,168-170}$ In conjunction, macrophages from $A h R^{-/-}$mice secreted higher IL-6, IL-1 $\beta$, and TNF- $\alpha$ levels in response to LPS than WT mice. ${ }^{132,169}$ AhR-dependent repression of IL-6 production following LPS stimulation in macrophages depends on two independent mechanisms: (i) suppression of histamine production via the histamine 1 receptor signaling ${ }^{169}$ and (ii) formation of an AhR-Stat1 complex inhibiting NF-kB transcriptional activity in their promoter region. ${ }^{171}$ AhR activation also enhances the expression of plasminogen activator inhibitor-2 (Pai-2), an inhibitor of caspase-1 activation, which suppresses the production of IL-1 $\beta$ in response to LPS treatment in macrophages. ${ }^{168}$

The protective effect of AhR activation is not restricted to LPS or Gram-negative bacteria-induced immunopathology. Rather, the same response is similarly observed for Gram-positive intestinal pathogens. Listeria monocytogenes, which is often used as model intracellular pathogen, is a facultative bacterium that causes foodborne disease, considerable morbidity and a relatively high mortality rate. AhR deficiency in mice results in higher susceptibility to $L$. monocytogenes, ${ }^{172}$ despite increased production of proinflammatory cytokines, such as IL- 6 and TNF- $a$, by macrophages after infection. ${ }^{113}$ Moreover, administration of FICZ protects against $L$. monocytogenes infection. ${ }^{171}$ Although AhR signaling is indispensable for optimal resistance to $L$. monocytogenes infection by improving macrophage survival and ROS production, ${ }^{171} \mathrm{AhR}$ is not essential for the adaptive immune response to $L$. monocytogenes. ${ }^{172}$

AhR is also involved in response to Clostridium difficile, which is a ubiquitous, anaerobic, spore-forming Gram-positive bacterium. Only toxigenic strains have pathogenic potential, and microbiota alterations after antibiotic use is a major risk factor for $C$. difficileassociated infection in humans. Supplementation of a semipurified diet with $\mathrm{I} 3 \mathrm{C}$ reduces the severity of $C$. difficile infection in WT and $A h R^{-1-}$ mice, suggesting that the effects of I3C may be mediated by both AhR-dependent and independent mechanisms. ${ }^{173}$ At least in WT mice, I3C diet-mediated protection against C. difficile infection was due to increased populations of cecal Treg, ILC3, $\gamma \delta \mathrm{T}$ cells, and neutrophils before infection. ${ }^{173}$

In addition to its protective effect against intestinal pathogenic bacteria, AhR is also involved in resistance to fungi. Administration of IAld, a bacteria-derived AhR ligand, to Candida albicans-infected WT mice induced IL-22 production by colonic NKp46 ${ }^{+}$cells, which enhanced resistance to fungi. These effects were mediated by $\mathrm{AhR}$, as they were lost in $A h R^{-/-}$mice. ${ }^{71}$ Colonization of conventional mice treated with antibiotics or GF mice by IAldproducing $L$. reuteri before $C$. albicans infection also improved resistance to fungi via induction of IL-22. ${ }^{71}$ Moreover, indole and its derivate indole-3-acetonitrile inhibited C. albicans biofilm formation in vitro, as well as C. albicans filamentation and attachment to IECs. ${ }^{174}$ These studies suggest that AhR activation by its ligands may be a potential therapeutic target for bacterial and fungal intestinal infection.

\section{AHR AND COLORECTAL CANCER}

Emerging evidence has indicated that $A h R$ also has an important role in intestinal tumorigenesis regulation. However, due to the pro- and anti-carcinogenic properties of $\mathrm{AhR}$, its role remains controversial. AhR deficiency in mice results in enhanced formation of spontaneous colonic polyps and cecal tumors. ${ }^{175,176}$ The suppression of intestinal tumor development by $A h R$ is dependent on $\beta$-catenin degradation and on apoptosis-associated speck-like protein containing a caspase recruitment protein (ASC), which has an essential role in caspase- 1 activation in inflammasomes, ${ }^{176}$ but not on the adenomatous polyposis coli system. ${ }^{176}$ Moreover, AhR regulates Pai-2 gene expression through a mechanism involving NF-KB to suppress caspase-1 activation, ${ }^{168}$ resulting in negative regulation of $\mathrm{IL}-1 \beta$ secretion. ${ }^{177}$ In $A h R^{-/-}$ mice, cecal tumorigenesis is decreased after blocking IL-1 $\beta$ signaling with a caspase- 1 inhibitor. ${ }^{55}$ Interestingly, no cecal tumors were observed in germ-free $A h R^{-/-}$mice, suggesting a key role of the gut microbiota in cecal tumor development in $A h R^{-/-}$ mice. ${ }^{176}$ Compared with conventional $A h R^{-/-}$mice, $A h R^{-/-} / A S C^{-1}$ double knockout mice exhibit reduced tumor development, suggesting that AhR acts as a tumor suppressor in inflammation-associated intestinal neoplasia. ${ }^{176}$ Compared with $A p c^{\mathrm{Min} /+}$ mice, which develop cecal and small intestinal tumors, $\mathrm{ApC}^{\mathrm{Min} /+} / \mathrm{AhR}^{+/-}$mice have increased tumor incidence, ${ }^{175}$ suggesting a tumor suppressor role of AhR in non-inflammatory intestinal carcinogenesis. AhR expression is elevated in tumor tissues from human colon cancer and $A p c^{\mathrm{Min} / \pm}$ mice compared with normal tissues. ${ }^{178}$ Moreover, small interfering RNA-mediated AhR knockdown in human colon cancer cell lines (HCT116 and HT29) leads to enhanced cell growth, whereas treatment with various AhR ligands, such as methylcholanthrene, I3C and DIM, inhibits cell proliferation, confirming that AhR acts as a tumor suppressor, at least partly through direct effects on tumor cells. ${ }^{178}$ In human and $\mathrm{ApC}^{\mathrm{Min} / \pm}$ mice colon cancer tissues AhR is localized to both stromal and tumors cells, whereas in normal intestines AhR is localized to stromal cells, the LP, and lymphoid follicles that contain immune cells, suggesting a potential role of AhR through effects on stromal cells. $^{178}$

In vitro, DIM and ICZ are also able to stimulate apoptosis in human colon LS-174 and Caco-2 adenocarcinoma cell lines but not in a non-transformed human colon epithelial cell line. ${ }^{179}$ Pretreatment with a combination of ICZ and isothiocyanate sulforaphane (SUL) reduced DNA damage in LS-174 cells treated with benzo $(a)$ pyrene or $\mathrm{H}_{2} \mathrm{O}_{2} \cdot{ }^{179}$ However, it remains to be proven whether protection against DNA damage conferred by ICZ and SUL is dependent on AhR. Diets containing AhR ligands, such as I3C and DIM, reduce the number and incidence of intestinal tumors by $\beta$-catenin degradation in $\mathrm{Apc}^{\mathrm{Min} /+}$ mice. ${ }^{175}$ Treatment 
with DIM also decreases the number of colon tumors in colitisassociated carcinogenesis induced by azoxymethane/DSS in WT mice. ${ }^{180}$ These studies all suggest that AhR has distinct roles in tumor suppression by regulating the gut immune system in normal tissues and negatively regulating tumor growth in the context of cancer.

Although these studies indicate that AhR ligands have anticarcinogenic properties, some studies have likewise demonstrated pro-carcinogenic effects of AhR ligands. Different studies have shown increased expression of AhR in tumor cells of several human cancers, including prostate, ${ }^{181}$ lung, ${ }^{182}$ breast, ${ }^{183}$ pancreatic $^{184}$ and gastric cancer, ${ }^{185}$ and have designated AhR an anticancer target. The overexpression of CYP1A1 and CYP1B1 in human colon tumors also suggests activation of AhR. ${ }^{186}$ Moreover, expression of a constitutively active $A h R$ in transgenic mice was found to induce stomach tumors. ${ }^{187}$ However, AhR is expressed by other cell types, including immune cells, which have an anti-tumor role. Consequently, the effect of constitutive AhR expression on stomach tumor induction may also be indirectly mediated through signaling by other cell types. In contrast to the antiproliferative effect induced by the AhR agonists DIM and ICZ, ${ }^{178,179}$ TCDD induces phosphorylation of Src kinase, leading to activation of the epidermal growth factor receptor and downstream ERK1/2 signaling, which eventually enhances colon cancer cell proliferation. ${ }^{188}$ These in vitro results suggest that the pro- and anti-proliferative properties of AhR could be dependent on the AhR ligand used. Different growth factors, including TGF- $\beta$ and TNF- $a$, are downstream targets of AhR signaling pathways and thus could also be involved in the pro-proliferative effect of AhR. $^{23,186,189,190}$ In addition to these proliferative effects, AhR activation is involved in pro-carcinogenic effects by inducing expression of pro-inflammatory IL-1 $\beta$, matrix metalloproteinase-9 (MMP-9), and the BCRP/ABCG2 drug transporter in colon cancer cells. ${ }^{191}$ For instance, IL-1 $\beta$ is associated with the induction of MMP and is known to increase cancer cell growth and metastasis. ${ }^{192}$ In addition, increased expression of BCRP/ABCG2 has been observed in a number of cancer cells that develop drug resistance against chemotherapeutic compounds. ${ }^{193}$

Interestingly, most in vitro studies have shown that AhR ligands enhance colon cancer cell proliferation, whereas most in vivo studies have shown protective effects of AhR against intestinal cancer. These contradictory effects suggest that in vitro studies to determine the impact of AhR on colon carcinogenesis may not be appropriate; thus, further studies are needed to characterize the impact of AhR on colon carcinogenesis. Furthermore, the majority of in vitro studies have focused on the impact of AhR on cancer cells, whereas AhR is expressed by many cell types, including immune cells, which have a major anti-tumor role. Moreover, inflammation alone may lead to tumor formation. Consequently, AhR could also indirectly regulate colon carcinogenesis through its effects on immune cells. This could explain the differences observed between in vitro and in vivo results. Studies using hematopoietic chimeras or conditional knockout mice could be useful to determine, in vivo, whether the role of AhR in colon carcinogenesis is intrinsic to epithelial cells or is mediated by immune cells.

Collectively, despite some conflicting in vitro results, these data suggest that activation of the AhR pathway by dietary or microbiota-derived AhR agonists could be a target of preventive or therapeutic strategies against colorectal cancer.

\section{CONCLUSION}

AhR ligands, derived from food, intestinal microbiota, and environmental contaminants, are naturally enriched within the gastrointestinal tract. AhR is expressed by different cell types in the gut, including immune cells and IEC, and has a pivotal role in regulating a myriad of cellular host functions. It logically follows that AhR, especially when its activation is perturbed, could influence the progression and outcome of different diseases. Data from animal models, maintained under diets containing or deficient for AhR ligands, support the complex roles of AhR in the regulation of intestinal homeostasis, particularly in regard to immune aspects. Disruption of intestinal homeostasis has been described in different intestinal inflammatory diseases; available data from both animal models and mammalian cell lines suggest the remarkable potential of dietary-derived AhR agonists, indole derivatives and/or AhR ligand-producing probiotic organisms as preventive and therapeutic interventions. Modulating the AhR pathway is also an attractive curative strategy for other intestinal pathological conditions, such as infection and colorectal cancer. However, the origin and structure of AhR ligands are diverse and their effects are not similar, likely due to their differential receptor affinity and downstream signaling effects. Therefore, further studies are needed to better understand the complex role of AhR in the regulation of intestinal homeostasis, and safely and efficiently target it as a preventive and therapeutic approach.

\section{ACKNOWLEDGEMENTS}

HS received funding from the European Research Council (ERC) under the European Union's Horizon 2020 Research and Innovation Programme (ERC-2016-StG-71577) and from JPI HDHL Intestinal Microbiomics (ANR-15-HDIM-0001-1). Funding: HS: Consulting fee for Danone, MSD, Takeda, Abbvie, Astellas, Enterome, Maat, Novartis, BMS, Co-founder of Nextbiotix; BL and JMN: None.

\section{AUTHOR CONTRIBUTIONS}

$\mathrm{BL}$ and $\mathrm{JMN}$ reviewed the literature. HS coordinated the work and organized the writing. All the authors wrote the manuscript.

\section{ADDITIONAL INFORMATION}

Competing interests: The authors declare no competing interests.

Publisher's note: Springer Nature remains neutral with regard to jurisdictional claims in published maps and institutional affiliations.

\section{REFERENCES}

1. Gu, Y. Z., Hogenesch, J. B. \& Bradfield, C. A. The PAS superfamily: sensors of environmental and developmental signals. Annu. Rev. Pharmacol. Toxicol. 40, 519-561 (2000).

2. Mandal, P. K. Dioxin: a review of its environmental effects and its aryl hydrocarbon receptor biology. J. Comp. Physiol. B, Biochem. Syst. Environ. Physiol. 175, 221-230 (2005).

3. Frericks, M., Meissner, M. \& Esser, C. Microarray analysis of the AHR system: tissue-specific flexibility in signal and target genes. Toxicol. Appl. Pharmacol. 220, 320-332 (2007).

4. Uhlen, M. et al. Towards a knowledge-based Human Protein Atlas. Nat. Biotechnol. 28, 1248-1250 (2010).

5. Hankinson, O. The aryl hydrocarbon receptor complex. Annu. Rev. Pharmacol. Toxicol. 35, 307-340 (1995).

6. Schmidt, J. V. \& Bradfield, C. A. Ah receptor signaling pathways. Annu. Rev. Cell. Dev. Biol. 12, 55-89 (1996).

7. Denison, M. S. \& Nagy, S. R. Activation of the aryl hydrocarbon receptor by structurally diverse exogenous and endogenous chemicals. Annu. Rev. Pharmacol. Toxicol. 43, 309-334 (2003).

8. Denison, M. S. \& Heath-Pagliuso, S. The Ah receptor: a regulator of the biochemical and toxicological actions of structurally diverse chemicals. Bull. Environ. Contam. Toxicol. 61, 557-568 (1998).

9. Kazlauskas, A., Poellinger, L. \& Pongratz, I. Evidence that the co-chaperone p23 regulates ligand responsiveness of the dioxin (Aryl hydrocarbon) receptor. J. Biol. Chem. 274, 13519-13524 (1999).

10. Hord, N. G. \& Perdew, G. H. Physicochemical and immunocytochemical analysis of the aryl hydrocarbon receptor nuclear translocator: characterization of two monoclonal antibodies to the aryl hydrocarbon receptor nuclear translocator. Mol. Pharmacol. 46, 618-626 (1994).

11. Probst, M. R., Reisz-Porszasz, S., Agbunag, R. V., Ong, M. S. \& Hankinson, O. Role of the aryl hydrocarbon receptor nuclear translocator protein in aryl hydrocarbon (dioxin) receptor action. Mol. Pharmacol. 44, 511-518 (1993). 
12. Roberts, B. J. \& Whitelaw, M. L. Degradation of the basic helix-loop-helix/PerARNT-Sim homology domain dioxin receptor via the ubiquitin/proteasome pathway. J. Biol. Chem. 274, 36351-36356 (1999).

13. Friling, R. S., Bensimon, A., Tichauer, Y. \& Daniel, V. Xenobiotic-inducible expression of murine glutathione S-transferase Ya subunit gene is controlled by an electrophile-responsive element. Proc Natl Acad Sci USA 87, 6258-6262 (1990).

14. Brauze, D. et al. Diversified expression of aryl hydrocarbon receptor dependent genes in human laryngeal squamous cell carcinoma cell lines treated with $\beta$ naphthoflavone. Toxicol. Lett. 231, 99-107 (2014).

15. Ma, Q., Kinneer, K., Bi, Y., Chan, J. Y. \& Kan, Y. W. Induction of murine NAD(P)H: quinone oxidoreductase by 2,3,7,8-tetrachlorodibenzo-p-dioxin requires the CNC (cap ' $n$ ' collar) basic leucine zipper transcription factor Nrf2 (nuclear factor erythroid 2-related factor 2): cross-interaction between AhR (aryl hydrocarbon receptor) and Nrf2 signal transduction. Biochem. J. 377, 205-213 (2004).

16. Auyeung, D. J., Kessler, F. K. \& Ritter, J. K. Mechanism of rat UDPglucuronosyltransferase $1 \mathrm{~A} 6$ induction by oltipraz: evidence for a contribution of the Aryl hydrocarbon receptor pathway. Mol. Pharmacol. 63, 119-127 (2003).

17. Dunn, T. J., Lindahl, R. \& Pitot, H. C. Differential gene expression in response to 2,3,7,8-tetrachlorodibenzo-p-dioxin (TCDD). Noncoordinate regulation of a TCDD-induced aldehyde dehydrogenase and cytochrome P-450c in the rat. J. Biol. Chem. 263, 10878-10886 (1988).

18. Stockinger, B., Meglio, P., Di, Gialitakis, M. \& Duarte, J. H. H. The aryl hydrocarbon receptor: multitasking in the immune system. Annu. Rev. Immunol. 32, 403-432 (2014).

19. Puga, A. et al. Aromatic hydrocarbon receptor interaction with the retinoblastoma protein potentiates repression of E2F-dependent transcription and cell cycle arrest. J. Biol. Chem. 275, 2943-2950 (2000).

20. Frueh, F. W., Hayashibara, K. C., Brown, P. O. \& Whitlock, J. P. Use of cDNA microarrays to analyze dioxin-induced changes in human liver gene expression. Toxicol. Lett. 122, 189-203 (2001).

21. Boverhof, D. R. et al. Temporal and dose-dependent hepatic gene expression patterns in mice provide new insights into TCDD-Mediated hepatotoxicity. Toxicol. Sci. 85, 1048-1063 (2005).

22. Tijet, $\mathrm{N}$. et al. Aryl hydrocarbon receptor regulates distinct dioxin-dependent and dioxin-independent gene batteries. Mol. Pharmacol. 69, 140-153 (2006).

23. Puga, A., Ma, C. \& Marlowe, J. L. The aryl hydrocarbon receptor cross-talks with multiple signal transduction pathways. Biochem. Pharmacol. 77, 713-722 (2009).

24. Vogel, C. F., Sciullo, E. \& Matsumura, F. Involvement of RelB in aryl hydrocarbon receptor-mediated induction of chemokines. Biochem. Biophys. Res. Commun. 363, 722-726 (2007).

25. Tian, Y. Ah receptor and NF-kappaB interplay on the stage of epigenome. Biochem. Pharmacol. 77, 670-680 (2009).

26. Ohtake, F., Fujii-Kuriyama, Y. \& Kato, S. AhR acts as an E3 ubiquitin ligase to modulate steroid receptor functions. Biochem. Pharmacol. 77, 474-484 (2009).

27. Wilson, S. R., Joshi, A. D. \& Elferink, C. J. The tumor suppressor Kruppel-like factor 6 is a novel aryl hydrocarbon receptor DNA binding partner. J. Pharmacol. Exp. Ther. 345, 419-429 (2013).

28. Dang, D. T., Pevsner, J. \& Yang, V. W. The biology of the mammalian Krüppel-like family of transcription factors. Int. J. Biochem. Cell. Biol. 32, 1103-1121 (2000).

29. Andreoli, V., Gehrau, R. C. \& Bocco, J. L. Biology of Krüppel-like factor 6 transcriptional regulator in cell life and death. IUBMB. Life. 62, 896-905 (2010).

30. Narla, G. et al. In vivo regulation of p21 by the Kruppel-like factor 6 tumorsuppressor gene in mouse liver and human hepatocellular carcinoma. Oncogene. 26, 4428-4434 (2007).

31. Sangodkar, J. et al. Functional role of the KLF6 tumour suppressor gene in gastric cancer. Eur. J. Cancer. 45, 666-676 (2009).

32. Reeves, H. L. et al. Kruppel-like factor 6 (KLF6) is a tumor-suppressor gene frequently inactivated in colorectal cancer. Gastroenterology. 126, 1090-1103 (2004).

33. Bureau, C. et al. Expression and Function of Kruppel Like-Factors (KLF) in Carcinogenesis. Curr. Genomics. 10, 353-360 (2009).

34. Jackson, D. P., Li, H., Mitchell, K. A., Joshi, A. D. \& Elferink, C. J. Ah receptormediated suppression of liver regeneration through NC-XRE-driven p21Cip1 expression. Mol. Pharmacol. 85, 533-541 (2014).

35. Gillner, M. et al. Interactions of indolo[3,2-b]carbazoles and related polycyclic aromatic hydrocarbons with specific binding sites for 2,3,7,8-tetrachlorodibenzo-p-dioxin in rat liver. Mol. Pharmacol. 44, 336-345 (1993).

36. Kafafi, S. A., Afeefy, H. Y., Said, H. K. \& Kafafi, A. G. Relationship between aryl hydrocarbon receptor binding, induction of aryl hydrocarbon hydroxylase and 7-ethoxyresorufin O-deethylase enzymes, and toxic activities of aromatic xenobiotics in animals. A new model. Chem. Res. Toxicol. 6, 328-334 (1993).

37. Denison, M. S., Phelan, D., Winter, G. M. \& Ziccardi, M. H. Carbaryl, a carbamate insecticide, is a ligand for the hepatic Ah (dioxin) receptor. Toxicol. Appl. Pharmacol. 152, 406-414 (1998).
38. Poland, A. \& Knutson, J. C. 2,3,7,8-tetrachlorodibenzo-p-dioxin and related halogenated aromatic hydrocarbons: examination of the mechanism of toxicity. Annu. Rev. Pharmacol. Toxicol. 22, 517-554 (1982).

39. Wattenberg, L. W. \& Loub, W. D. Inhibition of polycyclic aromatic hydrocarboninduced neoplasia by naturally occurring indoles. Cancer. Res. 38, 1410-1413 (1978).

40. Bjeldanes, L. F., Kim, J. Y., Grose, K. R., Bartholomew, J. C. \& Bradfield, C. A. Aromatic hydrocarbon responsiveness-receptor agonists generated from indole-3-carbinol in vitro and in vivo: comparisons with 2,3,7,8-tetrachlorodibenzo-p-dioxin. Proc Natl Acad Sci USA 88, 9543-9547 (1991).

41. Loub, W. D., Wattenberg, L. W. \& Davis, D. W. Aryl hydrocarbon hydroxylase induction in rat tissues by naturally occurring indoles of cruciferous plants. $J$. Natl. Cancer. Inst. 54, 985-988 (1975).

42. Shapiro, T. A., Fahey, J. W., Wade, K. L., Stephenson, K. K. \& Talalay, P. Chemoprotective glucosinolates and isothiocyanates of broccoli sprouts: metabolism and excretion in humans. Cancer. Epidemiol. Biomarkers. Prev. 10, 501-508 (2001).

43. Ito, S., Chen, C., Satoh, J., Yim, S. \& Gonzalez, F. J. Dietary phytochemicals regulate whole-body CYP1A1 expression through an arylhydrocarbon receptor nuclear translocator-dependent system in gut. J. Clin. Invest. 117, 1940-1950 (2007).

44. Gillner, M., Bergman, J., Cambillau, C. \& Gustafsson, J. A. Interactions of rutaecarpine alkaloids with specific binding sites for 2,3,7,8-tetrachlorodibenzo-pdioxin in rat liver. Carcinogenesis. 10, 651-654 (1989).

45. Gillner, M., Bergman, J., Cambillau, C., Fernström, B. \& Gustafsson, J. A. Interactions of indoles with specific binding sites for 2,3,7,8-tetrachlorodibenzo-pdioxin in rat liver. Mol. Pharmacol. 28, 357-363 (1985).

46. MacDonald, C. J., Ciolino, H. P. \& Yeh, G. C. Dibenzoylmethane modulates aryl hydrocarbon receptor function and expression of cytochromes P50 1A1, 1A2, and 1B1. Cancer. Res. 61, 3919-3924 (2001).

47. Ciolino, H. P., Daschner, P. J., Wang, T. T. \& Yeh, G. C. Effect of curcumin on the aryl hydrocarbon receptor and cytochrome P450 1A1 in MCF-7 human breast carcinoma cells. Biochem. Pharmacol. 56, 197-206 (1998).

48. Ashida, H. Suppressive effects of flavonoids on dioxin toxicity. Biofactors. 12, 201-206 (2000).

49. Yannai, S., Day, A. J., Williamson, G. \& Rhodes, M. J. Characterization of flavonoids as monofunctional or bifunctional inducers of quinone reductase in murine hepatoma cell lines. Food. Chem. Toxicol. 36, 623-630 (1998).

50. Ashida, H., Fukuda, I., Yamashita, T. \& Kanazawa, K. Flavones and flavonols at dietary levels inhibit a transformation of aryl hydrocarbon receptor induced by dioxin. FEBS Lett. 476, 213-217 (2000).

51. Ciolino, H. P., Wang, T. T. \& Yeh, G. C. Diosmin and diosmetin are agonists of the aryl hydrocarbon receptor that differentially affect cytochrome P450 1A1 activity. Cancer. Res. 58, 2754-2760 (1998).

52. Canivenc-Lavier, M. C. et al. Comparative effects of flavonoids and model inducers on drug-metabolizing enzymes in rat liver. Toxicology. 114, 19-27 (1996).

53. Amakura, Y. et al. Preliminary screening of the inhibitory effect of food extracts on activation of the aryl hydrocarbon receptor induced by 2,3,7,8-tetrachlorodibenzo-p-dioxin. Biol. Pharm. Bull. 25, 272-274 (2002).

54. Paganga, G. \& Rice-Evans, C. A. The identification of flavonoids as glycosides in human plasma. FEBS Lett. 401, 78-82 (1997).

55. Schmidt, J. V., Su, G. H., Reddy, J. K., Simon, M. C. \& Bradfield, C. A. Characterization of a murine Ahr null allele: involvement of the Ah receptor in hepatic growth and development. Proc Natl Acad Sci USA 93, 6731-6736 (1996).

56. Lahvis, G. P. et al. Portosystemic shunting and persistent fetal vascular structures in aryl hydrocarbon receptor-deficient mice. Proc Natl Acad Sci USA 97, 10442-10447 (2000).

57. Perdew, G. H. \& Babbs, C. F. Production of Ah receptor ligands in rat fecal suspensions containing tryptophan or indole-3-carbinol. Nutr. Cancer. 16, 209-218 (1991).

58. Bradfield, C. A. \& Bjeldanes, L. F. Modification of carcinogen metabolism by indolylic autolysis products of Brassica oleraceae. Adv. Exp. Med. Biol. 289, 153-163 (1991).

59. Kruif, C. A. De et al. Structure elucidation of acid reaction products of indole-3carbinol: detection in vivo and enzyme induction in vitro. Chem. Biol. Interact. 80, 303-315 (1991).

60. Jellinck, P. H. et al. Ah receptor binding properties of indole carbinols and induction of hepatic estradiol hydroxylation. Biochem. Pharmacol. 45, 1129-1136 (1993).

61. Paine, A. J. \& Francis, J. E. The induction of benzo[a]pyrene-3-mono-oxygenase by singlet oxygen in liver cell culture is mediated by oxidation products of histidine. Chem. Biol. Interact. 30, 343-353 (1980)

62. Paine, A. J. Induction of benzo[a]pyrene Mono-oxygenase in liver cell culture by the photochemical generation of active oxygen species. Evidence for the 
involvement of singlet oxygen and the formation of a stable inducing intermediate. Biochem. J. 158, 109-117 (1976).

63. Goerz, G., Merk, H., Bolsen, K., Tsambaos, D. \& Berger, H. Influence of chronic UVlight exposure on hepatic and cutaneous monooxygenases. Experientia. 39, 385-386 (1983).

64. Goerz, G. et al. Influence of UVA and UVB irradiation on hepatic and cutaneous P450 isoenzymes. Arch. Dermatol. Res. 289, 46-51 (1996).

65. Rannug, U. et al. Structure elucidation of two tryptophan-derived, high affinity Ah receptor ligands. Chem. Biol. 2, 841-845 (1995).

66. Rannug, A. et al. Certain photooxidized derivatives of tryptophan bind with very high affinity to the Ah receptor and are likely to be endogenous signal substances. J. Biol. Chem. 262, 15422-15427 (1987).

67. Rannug, A. \& Fritsche, E. The aryl hydrocarbon receptor and light. Biol. Chem. 387, 1149-1157 (2006).

68. Yanofsky, C., Horn, V. \& Gollnick, P. Physiological studies of tryptophan transport and tryptophanase operon induction in Escherichia coli. J. Bacteriol. 173, 6009-6017 (1991).

69. Fujioka, M. \& Wada, H. The bacterial oxidation of indole. Biochim. Biophys. Acta. 158, 70-78 (1968).

70. Berry, D. F., Madsen, E. L. \& Bollag, J. M. Conversion of indole to oxindole under methanogenic conditions. Appl. Environ. Microbiol. 53, 180-182 (1987).

71. Zelante, T., Rossana et al. Tryptophan catabolites from microbiota engage aryl hydrocarbon receptor and balance mucosal reactivity via interleukin-22. Immunity. 39, 372-385 (2013).

72. Cervantes-Barragan, L. et al. Lactobacillus reuteri induces gut intraepithelial CD4 + CD8aa + T cells. Science 357, 806-810 (2017).

73. Kawamura-Sato, K. et al. Role of multiple efflux pumps in Escherichia coli in indole expulsion. FEMS. Microbiol. Lett. 179, 345-352 (1999).

74. Piñero-Fernandez, S., Chimerel, C., Keyser, U. F. \& Summers, D. K. Indole transport across Escherichia coli membranes. J. Bacteriol. 193, 1793-1798 (2011).

75. Bansal, T., Alaniz, R. C., Wood, T. K. \& Jayaraman, A. The bacterial signal indole increases epithelial-cell tight-junction resistance and attenuates indicators of inflammation. Proc Natl Acad Sci USA 107, 228-233 (2010).

76. Whitfield-Cargile, C. M. et al. The microbiota-derived metabolite indole decreases mucosal inflammation and injury in a murine model of NSAID enteropathy. Gut Microbes 7, 246-261 (2016).

77. Fung, T. C., Olson, C. A. \& Hsiao, E. Y. Interactions between the microbiota, immune and nervous systems in health and disease. Nat. Neurosci. 20, 145-155 (2017).

78. Mezrich, J. D. et al. An interaction between kynurenine and the aryl hydrocarbon receptor can generate regulatory T cells. J. Immunol. 185, 3190-3198 (2010).

79. Bohár, Z., Toldi, J., Fülöp, F. \& Vécsei, L. Changing the face of kynurenines and neurotoxicity: therapeutic considerations. Int. J. Mol. Sci. 16, 9772-9793 (2015).

80. Han, Q., Robinson, H., Cai, T., Tagle, D. A. \& Li, J. Biochemical and structural properties of mouse kynurenine aminotransferase III. Mol. Cell. Biol. 29, 784-793 (2009).

81. DiNatale, B. C. et al. Kynurenic acid is a potent endogenous aryl hydrocarbon receptor ligand that synergistically induces interleukin-6 in the presence of inflammatory signaling. Toxicol. Sci. 115, 89-97 (2010).

82. Goasduff, T., Dréano, Y., Guillois, B., Ménez, J. F. \& Berthou, F. Induction of liver and kidney CYP1A1/1A2 by caffeine in rat. Biochem. Pharmacol. 52, 1915-1919 (1996).

83. Iba, M. M., Scholl, H., Fung, J., Thomas, P. E. \& Alam, J. Induction of pulmonary CYP1A1 by nicotine. Xenobiotica. 28, 827-843 (1998).

84. Kim, H., Reddy, S. \& Novak, R. F. 3-Methylcholanthrene and pyridine effects on CYP1A1 and CYP1A2 expression in rat renal tissue. Drug. Metab. Dispos. 23, 818-824 (1995).

85. Daujat, $M$. et al. Omeprazole, an inducer of human CYP1A1 and $1 A 2$, is not a ligand for the Ah receptor. Biochem. Biophys. Res. Commun. 188, 820-825 (1992).

86. Aix, L., Rey-Grobellet, X., Larrieu, G., Lesca, P. \& Galtier, P. Thiabendazole is an inducer of cytochrome P4501A1 in cultured rabbit hepatocytes. Biochem. Biophys. Res. Commun. 202, 1483-1489 (1994).

87. Daujat, M. et al. Induction of CYP1A1 gene by benzimidazole derivatives during Caco-2 cell differentiation. Evidence for an aryl-hydrocarbon receptor-mediated mechanism. Eur. J. Biochem. 237, 642-652 (1996).

88. Jeong, H. G., Lee, S. S., Kim, H. K. \& Yang, K. H. Murine Cyp1a-1 induction in mouse hepatoma Hepa-1C1C7 cells by myristicin. Biochem. Biophys. Res. Commun. 233, 619-622 (1997).

89. Marcus, C. B., Wilson, N. M., Jefcoate, C. R., Wilkinson, C. F. \& Omiecinski, C. J. Selective induction of cytochrome P450 isozymes in rat liver by $4-n$-alkylmethylenedioxybenzenes. Arch. Biochem. Biophys. 277, 8-16 (1990).

90. Delescluse, C. et al. Cytotoxic effects and induction of cytochromes P450 1A1/2 by insecticides, in hepatic or epidermal cells: binding capability to the Ah receptor. Toxicol. Lett. 96-97, 33-39 (1998).
91. Ledirac, N. et al. Carbaryl induces CYP1A1 gene expression in HepG2 and $\mathrm{HaCaT}$ cells but is not a ligand of the human hepatic Ah receptor. Toxicol. Appl. Pharmacol. 144, 177-182 (1997).

92. Sandoz, C., Lesca, P., Narbonne, J. F. \& Carpy, A. Molecular characteristics of carbaryl, a CYP1A1 gene inducer. Arch. Biochem. Biophys. 373, 275-280 (2000).

93. Hahn, M. E. Aryl hydrocarbon receptors: diversity and evolution. Chem. Biol. Interact. 141, 131-160 (2002).

94. Hahn, M. E. The aryl hydrocarbon receptor: a comparative perspective. Comp. Biochem. Physiol. C. Pharmacol. Toxicol. Endocrinol. 121, 23-53 (1998).

95. Hahn, M. E., Karchner, S. I., Shapiro, M. A. \& Perera, S. A. Molecular evolution of two vertebrate aryl hydrocarbon (dioxin) receptors (AHR1 and AHR2) and the PAS family. Proc Natl Acad Sci USA 94, 13743-13748 (1997).

96. Ramadoss, P. \& Perdew, G. H. Use of 2-azido-3-[125]]iodo-7,8-dibromodibenzo$\mathrm{p}$-dioxin as a probe to determine the relative ligand affinity of human versus mouse aryl hydrocarbon receptor in cultured cells. Mol. Pharmacol. 66, 129-136 (2004).

97. Murray, I. A., Patterson, A. D. \& Perdew, G. H. Aryl hydrocarbon receptor ligands in cancer: friend and foe. Nat. Rev. Cancer. 14, 801-814 (2014).

98. Flaveny, C. A., Murray, I. A. \& Perdew, G. H. Differential gene regulation by the human and mouse aryl hydrocarbon receptor. Toxicol. Sci. 114, 217-225 (2010).

99. Leavy, O. Mucosal immunology: the 'AHR diet' for mucosal homeostasis. Nat. Rev. Immunol. 11, 806 (2011).

100. Furue, M., Takahara, M., Nakahara, T. \& Uchi, H. Role of AhR/ARNT system in skin homeostasis. Arch. Dermatol. Res. 306, 769-779 (2014).

101. Hao, N. \& Whitelaw, M. L. The emerging roles of AhR in physiology and immunity. Biochem. Pharmacol. 86, 561-570 (2013).

102. Han, B. et al. Aryl Hydrocarbon Receptor Activation in Intestinal Obstruction Ameliorates Intestinal Barrier Dysfunction Via Suppression of MLCK-MLC Phosphorylation Pathway. Shock. 46, 319-328 (2016).

103. Chinen, I. et al. The aryl hydrocarbon receptor/microRNA-212/132 axis in T cells regulates IL-10 production to maintain intestinal homeostasis. Int. Immunol. 27, 405-415 (2015).

104. Sonnenberg, G. F., Fouser, L. A. \& Artis, D. Border patrol: regulation of immunity, inflammation and tissue homeostasis at barrier surfaces by IL-22. Nat. Immunol. 12, 383-390 (2011).

105. Stelter, C. et al. Salmonella-induced mucosal lectin Reglll $\beta$ kills competing gut microbiota. PLoS. ONE. 6, e20749 (2011).

106. Schiering, C. et al. Feedback control of AHR signalling regulates intestinal immunity. Nature 542, 242-245 (2017).

107. Kiss, E. A. et al. Natural aryl hydrocarbon receptor ligands control organogenesis of intestinal lymphoid follicles. Science 334, 1561-1565 (2011).

108. Chimerel, C. et al. Bacterial metabolite indole modulates incretin secretion from intestinal enteroendocrine L cells. Cell Rep 9, 1202-1208 (2014).

109. Chiba, T., Chihara, J. \& Furue, M. Role of the Arylhydrocarbon Receptor (AhR) in the Pathology of Asthma and COPD. J Allergy (Cairo) 2012, 372384 (2012).

110. Li, Y. et al. Exogenous stimuli maintain intraepithelial lymphocytes via aryl hydrocarbon receptor activation. Cell 147, 629-640 (2011).

111. Stockinger, B. \& Omenetti, S. The dichotomous nature of T helper 17 cells. Nat. Rev. Immunol. 17, 535-544 (2017).

112. Qiu, J. et al. Group 3 innate lymphoid cells inhibit T-cell-mediated intestinal inflammation through aryl hydrocarbon receptor signaling and regulation of microflora. Immunity. 39, 386-399 (2013).

113. Kimura, A. et al. Aryl hydrocarbon receptor in combination with Stat 1 regulates LPS-induced inflammatory responses. J. Exp. Med. 206, 2027-2035 (2009).

114. Nguyen, N. T. et al. Aryl hydrocarbon receptor negatively regulates dendritic cell immunogenicity via a kynurenine-dependent mechanism. Proc Natl Acad Sci USA 107, 19961-19966 (2010).

115. Quintana, F. J. et al. An endogenous aryl hydrocarbon receptor ligand acts on dendritic cells and $T$ cells to suppress experimental autoimmune encephalomyelitis. Proc Natl Acad Sci USA 107, 20768-20773 (2010).

116. Zindl, C. L. et al. IL-22-producing neutrophils contribute to antimicrobial defense and restitution of colonic epithelial integrity during colitis. Proc Natl Acad Sci USA 110, 12768-12773 (2013).

117. Chen, F. et al. mTOR Mediates IL-23 Induction of Neutrophil IL-17 and IL-22 Production. J. Immunol. 196, 4390-4399 (2016).

118. Qiu, J. et al. The aryl hydrocarbon receptor regulates gut immunity through modulation of innate lymphoid cells. Immunity. 36, 92-104 (2012).

119. Wang, $X$. et al. Interleukin-22 alleviates metabolic disorders and restores mucosal immunity in diabetes. Nature 514, 237-241 (2014).

120. Rutz, S., Eidenschenk, C. \& Ouyang, W. IL-22, not simply a Th17 cytokine. Immunol. Rev. 252, 116-132 (2013).

121. Rescigno, M. Intestinal dendritic cells. Adv. Immunol. 107, 109-138 (2010).

122. Schiavi, E., Smolinska, S. \& O'Mahony, L. Intestinal dendritic cells. Curr. Opin. Gastroenterol. 31, 98-103 (2015). 
123. Liu, H., Ramachandran, I. \& Gabrilovich, D. I. Regulation of plasmacytoid dendritic cell development in mice by aryl hydrocarbon receptor. Immunol. Cell. Biol. 92, 200-203 (2014).

124. Goudot, C. et al. Aryl Hydrocarbon Receptor Controls Monocyte Differentiation into Dendritic Cells versus Macrophages. Immunity. 47, 582-596 (2017). e6.

125. Chng, S. H. et al. Ablating the aryl hydrocarbon receptor (AhR) in CD11c + cells perturbs intestinal epithelium development and intestinal immunity. Sci. Rep. 6, 23820 (2016).

126. Lee, J. S. et al. AHR drives the development of gut ILC22 cells and postnatal lymphoid tissues via pathways dependent on and independent of Notch. Nat. Immunol. 13, 144-151 (2011).

127. Kinnebrew, M. A. et al. Interleukin 23 production by intestinal CD103(+)CD11b $(+)$ dendritic cells in response to bacterial flagellin enhances mucosal innate immune defense. Immunity. 36, 276-287 (2012).

128. Rendon, J. L. et al. The role of aryl hydrocarbon receptor in interleukin-23dependent restoration of interleukin-22 following ethanol exposure and burn injury. Ann. Surg. 259, 582-590 (2014).

129. Siegemund, S. et al. Differential IL-23 requirement for IL-22 and IL-17A production during innate immunity against Salmonella enterica serovar Enteritidis. Int. Immunol. 21, 555-565 (2009).

130. Muñoz, M. et al. Interleukin (IL)-23 mediates Toxoplasma gondii-induced immunopathology in the gut via matrixmetalloproteinase- 2 and IL-22 but independent of IL-17. J. Exp. Med. 206, 3047-3059 (2009).

131. Veldhoen, M. et al. The aryl hydrocarbon receptor links TH17-cell-mediated autoimmunity to environmental toxins. Nature 453, 106-109 (2008).

132. Kimura, A., Naka, T., Nohara, K., Fujii-Kuriyama, Y. \& Kishimoto, T. Aryl hydrocarbon receptor regulates Stat1 activation and participates in the development of Th17 cells. Proc Natl Acad Sci USA 105, 9721-9726 (2008).

133. Veldhoen, M., Hirota, K., Christensen, J., O'Garra, A. \& Stockinger, B. Natural agonists for aryl hydrocarbon receptor in culture medium are essential for optimal differentiation of Th17 T cells. J. Exp. Med. 206, 43-49 (2009).

134. Lee, Y. et al. Induction and molecular signature of pathogenic TH17 cells. Nat Immunol. 13, 991-999 (2012).

135. Brandstätter, O. et al. Balancing intestinal and systemic inflammation through cell type-specific expression of the aryl hydrocarbon receptor repressor. Sci. Rep. 6, 26091 (2016).

136. Cui, G. et al. Liver $X$ receptor ( $L X R)$ mediates negative regulation of mouse and human Th17 differentiation. J. Clin. Invest. 121, 658-670 (2011).

137. Rutz, S. et al. Transcription factor c-Maf mediates the TGF- $\beta$-dependent suppression of IL-22 production in T(H)17 cells. Nat. Immunol. 12, 1238-1245 (2011).

138. Yeste, A. et al. IL-21 induces IL-22 production in CD4 + T cells. Nat. Commun. $\mathbf{5}$, 3753 (2014)

139. Quintana, F. J. et al. Control of $T(r e g)$ and $T(H) 17$ cell differentiation by the aryl hydrocarbon receptor. Nature 453, 65-71 (2008).

140. Liang, S. C. et al. Interleukin (IL)-22 and IL-17 are coexpressed by Th17 cells and cooperatively enhance expression of antimicrobial peptides. J. Exp. Med. 203 2271-2279 (2006)

141. Elizondo, G., Rodríguez-Sosa, M., Estrada-Muñiz, E., Gonzalez, F. J. \& Vega, L. Deletion of the aryl hydrocarbon receptor enhances the inflammatory response to Leishmania major infection. Int. J. Biol. Sci. 7, 1220-1229 (2011).

142. Goettel, J. A. et al. AHR Activation Is Protective against Colitis Driven by T Cells in Humanized Mice. Cell Rep 17, 1318-1329 (2016).

143. Gandhi, R. et al. Activation of the aryl hydrocarbon receptor induces human type 1 regulatory T cell-like and Foxp3(+) regulatory T cells. Nat. Immunol. 11, 846-853 (2010).

144. Funatake, C. J. et al. Expression of constitutively-active aryl hydrocarbon receptor in T-cells enhances the down-regulation of CD62L, but does not alter expression of $\mathrm{CD} 25$ or suppress the allogeneic CTL response. J. Immunotoxicol. 6, 194-203 (2009)

145. Saraiva, M. \& O'Garra, A. The regulation of IL-10 production by immune cells. Nat. Rev. Immunol. 10, 170-181 (2010).

146. Apetoh, L. et al. The aryl hydrocarbon receptor interacts with c-Maf to promote the differentiation of type 1 regulatory T cells induced by IL-27. Nat. Immunol. 11, 854-861 (2010).

147. Gagliani, N. et al. Th17 cells transdifferentiate into regulatory T cells during resolution of inflammation. Nature 523, 221-225 (2015).

148. Monteleone, I. et al. Aryl hydrocarbon receptor-induced signals up-regulate IL22 production and inhibit inflammation in the gastrointestinal tract. Gastroenterology. 141, 237-248 (2011). 248.e1.

149. Long, $X$. et al. NK cells contribute to persistent airway inflammation and AHR during the later stage of RSV infection in mice. Med. Microbiol. Immunol. 205, 459-470 (2016).

150. Zhao, Y. et al. MicroRNA-124 Promotes Intestinal Inflammation by Targeting Aryl Hydrocarbon Receptor in Crohn's Disease. J. Crohns Colitis 10, 703-712 (2016).
151. Li, J., Doty, A. \& Glover, S. C. Aryl hydrocarbon receptor signaling involves in the human intestinal ILC3/ILC1 conversion in the inflamed terminal ileum of Crohn's disease patients. Inflamm Cell Signal 3, e1404 (2016).

152. Lamas, B. et al. CARD9 impacts colitis by altering gut microbiota metabolism of tryptophan into aryl hydrocarbon receptor ligands. Nat. Med. 22, 598-605 (2016).

153. Furumatsu, K. et al. A role of the aryl hydrocarbon receptor in attenuation of colitis. Dig. Dis. Sci. 56, 2532-2544 (2011).

154. Takamura, T. et al. Activation of the aryl hydrocarbon receptor pathway may ameliorate dextran sodium sulfate-induced colitis in mice. Immunol. Cell. Biol. 88, 685-689 (2010).

155. Singh, N. P. et al. Activation of aryl hydrocarbon receptor (AhR) leads to reciprocal epigenetic regulation of FoxP3 and IL-17 expression and amelioration of experimental colitis. PLOS. ONE. 6, e23522 (2011).

156. Ivanov, S. \& Lindén, A. Interleukin-17 as a drug target in human disease. Trends. Pharmacol. Sci. 30, 95-103 (2009).

157. Gaboriau-Routhiau, V. et al. The key role of segmented filamentous bacteria in the coordinated maturation of gut helper $\mathrm{T}$ cell responses. Immunity. 31, 677-689 (2009)

158. Kumar, P. et al. Intestinal Interleukin-17 Receptor Signaling Mediates Reciprocal Control of the Gut Microbiota and Autoimmune Inflammation. Immunity. 44, 659-671 (2016)

159. Abildgaard, A., Elfving, B., Hokland, M., Wegener, G. \& Lund, S. Probiotic treatment reduces depressive-like behaviour in rats independently of diet. Psychoneuroendocrinology. 79, 40-48 (2017).

160. Fukumoto, $\mathrm{S}$. et al. Identification of a probiotic bacteria-derived activator of the aryl hydrocarbon receptor that inhibits colitis. Immunol. Cell. Biol. 92, 460-465 (2014).

161. Daniel, H. et al. High-fat diet alters gut microbiota physiology in mice. ISME. J. 8, 295-308 (2014)

162. Lamas, B. et al. Card9 mediates susceptibility to intestinal pathogens through microbiota modulation and control of bacterial virulence. Gut (2017).https://doi. org/10.1136/gutjnl-2017-314195

163. Hashimoto, T. et al. ACE2 links amino acid malnutrition to microbial ecology and intestinal inflammation. Nature 487, 477-481 (2012).

164. Shizuma, T., Mori, H. \& Fukuyama, N. Protective effect of tryptophan against dextran sulfate sodium- induced experimental colitis. Turk. J. Gastroenterol. 24, 30-35 (2013).

165. Islam, J. et al. Dietary tryptophan alleviates dextran sodium sulfate-induced colitis through aryl hydrocarbon receptor in mice. J. Nutr. Biochem. 42, 43-50 (2017).

166. Collins, J. W. et al. Citrobacter rodentium: infection, inflammation and the microbiota. Nat. Rev. Microbiol. 12, 612-623 (2014).

167. Guo, X. et al. Innate Lymphoid Cells Control Early Colonization Resistance against Intestinal Pathogens through ID2-Dependent Regulation of the Microbiota. Immunity. 42, 731-743 (2015).

168. Sekine, $H$. et al. Hypersensitivity of aryl hydrocarbon receptor-deficient mice to lipopolysaccharide-induced septic shock. Mol. Cell. Biol. 29, 6391-6400 (2009).

169. Masuda, K. et al. Aryl hydrocarbon receptor negatively regulates LPS-induced IL6 production through suppression of histamine production in macrophages. Int Immunol. 23, 637-645 (2011).

170. Bessede, A. et al. Aryl hydrocarbon receptor control of a disease tolerance defence pathway. Nature 511, 184-190 (2014).

171. Kimura, A. et al. Aryl hydrocarbon receptor protects against bacterial infection by promoting macrophage survival and reactive oxygen species production. Int. Immunol. 26, 209-220 (2014).

172. Shi, L. Z. et al. The aryl hydrocarbon receptor is required for optimal resistance to Listeria monocytogenes infection in mice. J. Immunol. 179, 6952-6962 (2007).

173. Julliard, W. et al. Amelioration of Clostridium difficile Infection in Mice by Dietary Supplementation With Indole-3-carbinol. Ann. Surg. 265, 1183-1191 (2017).

174. Oh, S., Go, G. W., Mylonakis, E. \& Kim, Y. The bacterial signalling molecule indole attenuates the virulence of the fungal pathogen Candida albicans. J. Appl. Microbiol. 113, 622-628 (2012).

175. Kawajiri, K. et al. Aryl hydrocarbon receptor suppresses intestinal carcinogenesis in ApcMin/+mice with natural ligands. Proc Natl Acad Sci USA 106, 13481-13486 (2009).

176. Ikuta, T. et al. ASC-associated inflammation promotes cecal tumorigenesis in aryl hydrocarbon receptor-deficient mice. Carcinogenesis. 34, 1620-1627 (2013).

177. Greten, F. R. et al. NF-kappaB is a negative regulator of IL-1beta secretion as revealed by genetic and pharmacological inhibition of IKKbeta. Cell 130, 918-931 (2007).

178. Ikuta, T., Kurosumi, M., Yatsuoka, T. \& Nishimura, Y. Tissue distribution of aryl hydrocarbon receptor in the intestine: Implication of putative roles in tumor suppression. Exp. Cell. Res. 343, 126-134 (2016). 
179. Bonnesen, C., Eggleston, I. M. \& Hayes, J. D. Dietary indoles and isothiocyanates that are generated from cruciferous vegetables can both stimulate apoptosis and confer protection against DNA damage in human colon cell lines. Cancer. Res. 61, 6120-6130 (2001).

180. Kim, Y. H. et al. 3,3'-diindolylmethane attenuates colonic inflammation and tumorigenesis in mice. Inflamm. Bowel. Dis. 15, 1164-1173 (2009).

181. Richmond, O. et al. The aryl hydrocarbon receptor is constitutively active in advanced prostate cancer cells. PLOS. ONE. 9, e95058 (2014).

182. Lin, P. et al. Overexpression of aryl hydrocarbon receptor in human lung carcinomas. Toxicol. Pathol. 31, 22-30 (2003).

183. Zhao, S. et al. HER2 overexpression-mediated inflammatory signaling enhances mammosphere formation through up-regulation of aryl hydrocarbon receptor transcription. Cancer. Lett. 330, 41-48 (2013).

184. Koliopanos, A. et al. Increased arylhydrocarbon receptor expression offers a potential therapeutic target for pancreatic cancer. Oncogene. 21, 6059-6070 (2002).

185. Ma, J.-X. X. et al. Concurrent expression of aryl hydrocarbon receptor and CYP1A1 but not CYP1A1 Mspl polymorphism is correlated with gastric cancers raised in Dalian, China. Cancer. Lett. 240, 253-260 (2006).

186. Androutsopoulos, V. P. et al. Expression profile of CYP1A1 and CYP1B1 enzymes in colon and bladder tumors. PLOS. ONE. 8, e82487 (2013).

187. Andersson, $\mathrm{P}$. et al. A constitutively active dioxin/aryl hydrocarbon receptor induces stomach tumors. Proc Natl Acad Sci USA 99, 9990-9995 (2002).

188. Xie, G., Peng, Z. \& Raufman, J.-P. P. Src-mediated aryl hydrocarbon and epidermal growth factor receptor cross talk stimulates colon cancer cell proliferation. Am. J. Physiol. Gastrointest. Liver. Physiol. 302, G1006-G1015 (2012).

189. Haarmann-Stemmann, T., Bothe, H. \& Abel, J. Growth factors, cytokines and their receptors as downstream targets of arylhydrocarbon receptor (AhR) signaling pathways. Biochem. Pharmacol. 77, 508-520 (2009).

190. Gomez-Duran, A. et al. Fitting a xenobiotic receptor into cell homeostasis: how the dioxin receptor interacts with TGFbeta signaling. Biochem. Pharmacol. 77, 700-712 (2009).

191. Tompkins, L. M. et al. A novel xenobiotic responsive element regulated by aryl hydrocarbon receptor is involved in the induction of BCRP/ABCG2 in LS174T cells. Biochem. Pharmacol. 80, 1754-1761 (2010).

192. Egeblad, M. \& Werb, Z. New functions for the matrix metalloproteinases in cancer progression. Nat. Rev. Cancer. 2, 161-174 (2002).

193. Noguchi, K., Katayama, K., Mitsuhashi, J. \& Sugimoto, Y. Functions of the breast cancer resistance protein (BCRP/ABCG2) in chemotherapy. Adv. Drug. Deliv. Rev. 61, 26-33 (2009).
194. Roth, C. W., Hoch, J. A. \& DeMoss, R. D. Physiological studies of biosynthetic indole excretion in Bacillus alvei. J. Bacteriol. 106, 97-106 (1971).

195. Brazier, J. S. et al. Isolation and identification of Clostridium spp. from infections associated with the injection of drugs: experiences of a microbiological investigation team. J. Med. Microbiol 51, 985-9 (2002).

196. Elsden, S. R., Hilton, M. G. \& Waller, J. M. The end products of the metabolism of aromatic amino acids by Clostridia. Arch. Microbiol. 107, 283-8 (1976).

197. Voss, J. G. Differentiation of two groups of Corynebacterium acnes. J. Bacteriol. 101, 392-7 (1970).

198. Schleifer, K. H., Kilpper-Bälz, R., Kraus, J. \& Gehring, F. Relatedness and classification of Streptococcus mutans and 'mutans-like' streptococci. J. Dent. Res. 63, 1047-50 (1984).

199. Tannock, G. W. Characteristics of Bacteroides isolates from the cecum of conventional mice. Appl. Environ. Microbiol. 33, 745-50 (1977).

200. DeMoss, R. D. \& Moser, K. Tryptophanase in diverse bacterial species. J. Bacteriol. 98, 167-71 (1969).

201. Booth, E. V. \& McDonald, S. A new group of enterobacteria, possibly a new Citrobacter sp. J. Med. Microbiol. 4, 329-36 (1971).

202. Li, G. \& Young, K. D. Indole production by the tryptophanase TnaA in Escherichia coli is determined by the amount of exogenous tryptophan. Microbiology (Reading, Engl.) 159, 402-10 (2013).

203. Pickett, M. J. Methods for identification of flavobacteria. J. Clin. Microbiol. 27, 2309-15 (1989).

204. Langworth, B. F. Fusobacterium necrophorum: its characteristics and role as an animal pathogen. Bacteriol Rev 41, 373-90 (1977).

205. Kilian, M. A taxonomic study of the genus Haemophilus, with the proposal of a new species. J. Gen. Microbiol. 93, 9-62 (1976).

206. Liu, Y., Mee, B. J. \& Mulgrave, L. Identification of clinical isolates of indolepositive Klebsiella spp., including Klebsiella planticola, and a genetic and molecular analysis of their beta-lactamases. J. Clin. Microbiol. 35, 2365-9 (1997).

207. Rezwan, F., Lan, R. \& Reeves, P. R. Molecular basis of the indole-negative reaction in Shigella strains: extensive damages to the tha operon by insertion sequences. J. Bacteriol. 186, 7460-5 (2004).

208. Nuidate, T. et al. Role of Indole Production on Virulence of Vibrio cholerae Using Galleria mellonella Larvae Model. Indian J. Microbiol. 56, 368-74 (2016).

209. Dealler, S. F., Hawkey, P. M. \& Millar, M. R. Enzymatic degradation of urinary indoxyl sulfate by Providencia stuartii and Klebsiella pneumoniae causes the purple urine bag syndrome. J. Clin. Microbiol. 26, 2152-6 (1988).

210. Magiatis, P. et al. Malassezia yeasts produce a collection of exceptionally potent activators of the Ah (dioxin) receptor detected in diseased human skin. J. Invest. Dermatol. 133, 2023-30 (2013). 